\title{
NR. 19
}

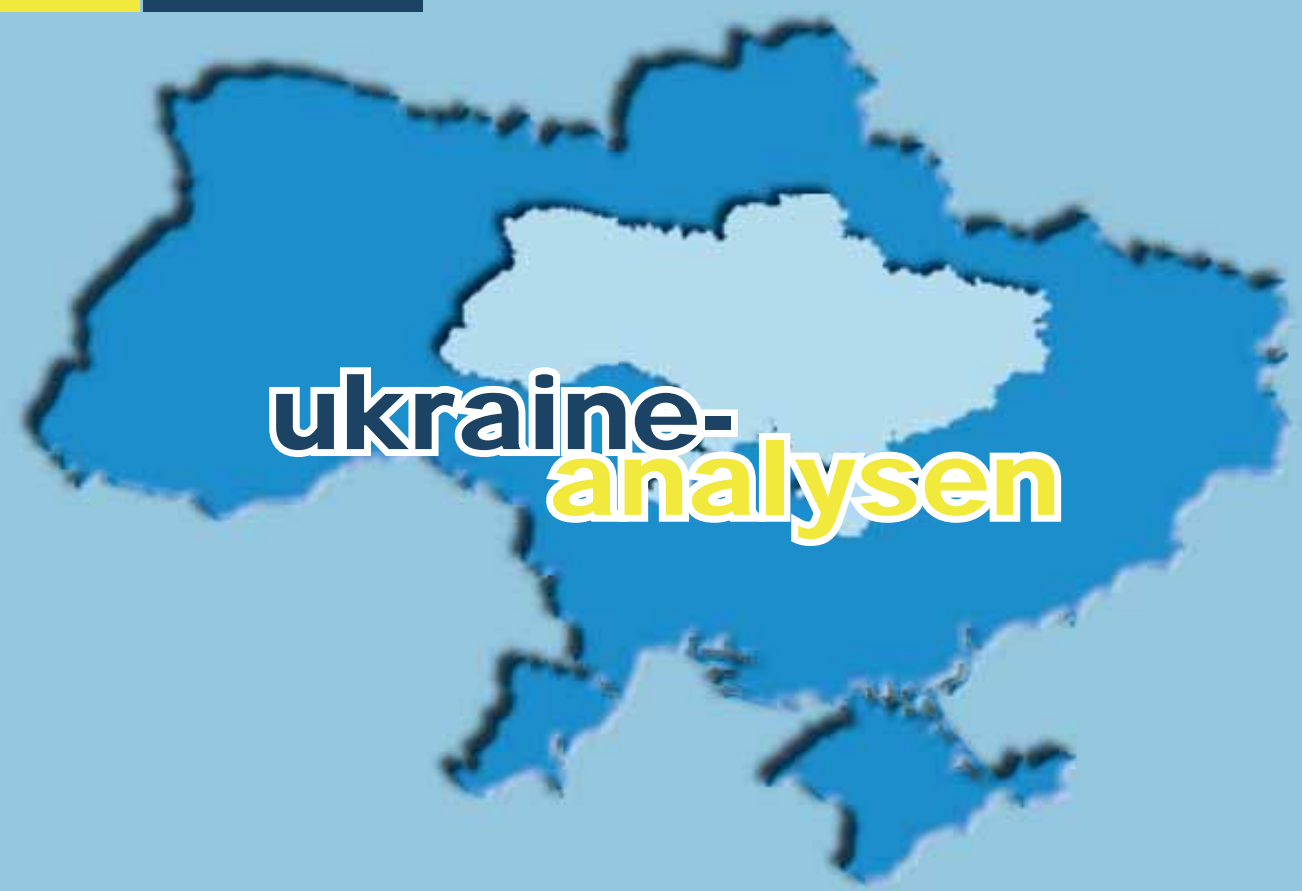

ukraine-analysen.de

\section{DER KONFLIKT ZWISCHEN REGIERUNG UND PRÄSIDENT DIE SPRACHENFRAGE}

ANALYSE

Man spielt mit den Regeln und nicht nach den Regeln.

Politische Ungewissheit in der Ukraine. Sarah Whitmore, Oxford

ANALYSE

Ukrainisch - Russisch: Sprachen, Sprachgebrauch, Sprachenkonflikte in der Ukraine 6 Gerhard Simon, Köln

TABELLEN UND GRAFIKEN ZUM TEXT

Statistiken und Umfragen zur Sprachenfrage

KOMMENTAR

Gespalten in Ost und West? Sprachenfrage und Geschichtspolitik in der Ukraine im Kontext der Wahlkämpfe 2004 und 2006. Wilfried Jilge, Leipzig

CHRONIK

Vom 22. Januar bis zum 13. Februar 2007

LESETIPP

Arbeitspapiere und Materialien Nr. 81. Das Comeback von Viktor Janukowitsch

LESETIPP

Abstracts der Artikel mit Ukraine-Bezug in OSTEUROPA 2-3/2007 


\title{
Analyse
}

\section{Man spielt mit den Regeln und nicht nach den Regeln. Politische Ungewissheit in der Ukraine}

\author{
Sarah Whitmore, Oxford Brookes University
}

\section{Zusammenfassung}

Der Rücktritt von Außenminister Boris Tarasjuk und das Inkrafttreten des Gesetzes „Über das Ministerkabinett" ohne die Unterschrift des Präsidenten sind die letzten Manifestationen des Machtkampfes innerhalb der Exekutive, der die Politik in Kiew seit dem Amtsantritt von Viktor Janukowitsch als Ministerpräsident im August 2006 dominiert hat. Auf einer Ebene ist dieser Machtkampf eine Reaktion auf gesetzliche Unstimmigkeiten und offensichtlichen Mängel der Verfassungsreform, die Präsident und Parlament jeweils zu ihren Gunsten korrigieren wollen. Der „Krieg der Gesetze“ spiegelt aber auch die post-sowjetische Verfasstheit an sich wider, in der formale politische Institutionen von den Akteuren als formbar und unverbindlich angesehen werden.

\section{Einleitung}

ie Parlamentswahlen im März 2006 führten sowohl zu einer Umgestaltung der politischen Kräfte als auch der formalen Spielregeln der Politik in der Ukraine. Die Partei der Regionen wurde zur größten Partei im Parlament und Viktor Janukowitsch gelang damit das Comeback als Ministerpräsident. Gleichzeitig trat die so genannte „Politreform“ in Kraft, d.h. die Verfassungsänderungen, die auf dem Höhepunkt der Orangen Revolution vereinbart worden waren. Die neue Regierung mit erweiterten Vollmachten wurde durch eine Koalition gebildet und nicht vom Präsidenten ernannt. Seitdem wird die ukrainische Politik durch erhöhte Unsicherheit und durch einen bitteren Machtkampf zwischen den politischen Eliten, vor allem zwischen Ministerpräsident und Präsident, gekennzeichnet.

\section{Offener Machtkampf}

S eit 1996 hat es ständig Versuche gegeben, die ukraiSnische Verfassung entweder direkt oder indirekt (durch Ermächtigungsgesetze) zu ändern. In dieser Hinsicht ist die heutige Unsicherheit nichts Neues. Die „Politreform“ mit dem gleichzeitigen Übergang zu einem proportionalen Wahlrecht führte zu den weit reichendsten Änderung des Machtssystems seit einem Jahrzehnt. Dies führte zu einer Übergangsperiode zu den neuen formalen Spielregeln, die sich in einem multidimensionalen Machtkampf manifestiert, der seinerseits den laufenden Kampf der Lobbyisten um wirtschaftliche Vorteile überlagert.

Ein hohes Maß an Unsicherheit hat dazu geführt, dass sich alle wesentlichen politischen Akteure noch mehr als zuvor auf kurzfristige anstatt auf strategische Ziele konzentrieren und dass die zynische Grundlage von politischen Deals und der legale Nihilismus, der ihre Handlungen leitet, offenbar werden. Die erbitterten Kämpfe um Personen (insbesondere die Entlassung von Ministern, die vom Präsidenten ernannt wurden) und Ämter (vor allem bei dem Gesetz „Über das Ministerkabinett") sind die deutlichsten Beispiele für diesen institutionellen Machtkampf.

\section{Startvorteil für Janukowitsch}

Seit seinem Amtsantritt als Ministerpräsident verS sucht Viktor Janukowitsch eindeutig, sich als mächtigster Politiker der Ukraine zu etablieren. Hierfür hat er substantielle Ressourcen: einen deutlichen Vorsprung in Meinungsumfragen als der Politiker der Ukraine, dem am meisten vertraut wird, die Unterstützung durch einige der reichsten Unternehmer der Ukraine und den Rückhalt durch eine Parlamentskoalition, in der seine Partei der Regionen mit 186 von 238 Sitzen mit Abstand die dominierende Kraft ist.

Trotz anfänglicher Vorhersagen, dass die „Antikrisenkoalition“ nur von kurzer Dauer sein würde, hat sie bis jetzt gehalten, da die Juniorpartner, die Sozialisten und Kommunisten, viel zu verlieren haben, falls sie die Koalition verlassen. Janukowitsch hat auch von dem schlechten Verhältnis zwischen den Blöcken außerhalb der regierenden Koalition profitiert, von denen jeder bedeutsame (Wirtschafts-)Anhänger hat, die sich nicht damit abfinden konnten, nicht an der Regierung beteiligt zu sein und damit keinen Zugang zu staatlichen Ressourcen zu haben.

Daher hatte Janukowitsch eine gute Position inne, um eine Politik durchzusetzen, die seine Position stärken würde und von der die wirtschaftlichen Interessen, die die Partei der Regionen unterstützen, profitieren würden. Obwohl es Spannungen zwischen den verschiede- 
nen Faktionen innerhalb der Partei gibt, sind diese im Vergleich zu Julia Timoschenkos Block und vor allem zu Unserer Ukraine von Juschtschenko eher gering.

Juschtschenko hat sich schwer damit getan, die neue konstitutionelle Realität und die neue Regierung zu akzeptieren, die beide nicht seinen Präferenzen entsprachen. Er schwankte zwischen Kompromiss und Widerstand.EingroßerKompromisswardie„Erklärung der nationalen Einheit", womit Juschtschenko die Ernennung von Juri Luzenko zum Innenminister und von vier Mitgliedern von Unserer Ukraine zu Justiz-, Jugend und Sport-, Kultur- und Gesundheitsministern durchsetzen konnte. Sein Widerstand zeigte sich durch den Gebrauch des Präsidentenvetos und die Anrufung des Verfassungsgerichts. Die Ressourcen des Präsidenten sind jedoch begrenzt durch die zahlenmäßige Unterlegenheit und die Zerstrittenheit der Parlamentsfraktion seiner Partei UnsereUkraine, durch seine geringe Popularität, die neuen Beschränkungen für seine konstitutionelle Autorität und beschränkte Unterstützung von Seiten seines Sekretariates, das durch hohe Personalfluktuation und unberechenbare Entlassungen (manchmal ganzer Abteilungen) durch das Staatsoberhaupt geschwächt ist.

\section{Konfliktpunkte}

$\Lambda$ nzeichen für einen grundlegenden Machtkampf Azwischen dem Präsidenten und dem Ministerpräsidenten gab es fast sofort nach der Ernennung der Regierung im August 2006, trotz der viel gepriesenen "Erklärung der nationalen Einheit", die eine koordinierte Politik der Exekutive auf den wichtigsten Gebieten sichern sollte, aber in Wahrheit als Feigenblatt diente, um Juschtschenko vor öffentlicher Demütigung durch die Ernennung von Janukowitsch zum Ministerpräsidenten zu bewahren. Janukowitsch ergriff die Initiative und forderte die Autorität des Präsident bei einer Reihe von politischen Themen und Prozedurfragen heraus, darunter bezüglich des Rechtes, den Verteidigungs- und den Außenminister zu entlassen (das nach der geänderten Verfassung ein Vorrecht des Präsidenten blieb) und legte einen Vorschlag für ein Gesetz „Über das Ministerkabinett“ vor, das lange überfällig war (nachdem Präsident Kutschma fünfmal sein Veto dagegen eingelegt hatte) und das die neue Gewaltenteilung eindeutig zugunsten des Ministerpräsidenten klarstellen würde.

Im Folgenden entzündeten sich Konflikte zwischen Ministerpräsident und Präsident an einer Vielzahl von Punkten, darunter das Recht des Präsidenten, Dekrete ohne die Gegenunterschrift des Ministerpräsidenten zu erlassen, die Gestaltung des Staatshaushaltes und die Rolle des Ministerpräsidenten in der Außenpolitik.
Der Einzug der fünf propräsidialen Minister in die RegierungJanukowitsch (zusätzlich zu den verfassungsmäßig gewährten Ernennungen des Verteidigungsund Außenministers) sollte den Weg dafür bahnen, dass Unsere Ukraine formal der Koalition beitrat, was das internationale Image von Janukowitsch verbessern und Unternehmern von Unsere Ukraine den Zugang zu staatlichen Ressourcen ermöglichen würde. Die rapide eskalierenden Spannungen zwischen Präsident und Ministerpräsident führten aber schnell dazu, dass Juschtschenkos Partei in die Opposition ging und ihre Minister aus der Regierung zurückzog.

\section{Der Streit um die Minister}

Die vier Minister von Unsere Ukraine traten im Oktober zurück, nicht jedoch Außenminister Boris Tarasjuk, der zusammen mit dem Parteilosen Anatoli Hrizenko gemäß der neuen Verfassung vom Präsidenten ernannt worden waren. Zusammen mit Innenminister Luzenko waren sie als Mitglieder der Regierungskoalition in der Lage die Regierungspolitik zu konterkarieren. Die pro-westliche Position von Tarasjuk stand im Konflikt zur Wahlkampfplattform der Partei der Regionen und hatte Russland schon lange geärgert. Es war bekannt, dass Luzenko Geschäftsleute in der Partei der Regionen mit Untersuchungen durch Rechtsschutzbehörden irritiert hatte. Das Verteidigungsministerium hat sehr viele Ressourcen, vor allem Land, unter seiner Kontrolle.

Es wurden Kampagnen lanciert, um die verbleibenden pro-Juschtschenko-Minister zu diskreditieren, indem sowohl Luzenko wie Hrizenko der Korruption beschuldigt wurden. Im Fall des Innenministers wurden sowohl eine parlamentarische als auch eine kriminalpolizeiliche Untersuchung durchgeführt, während die Rechnungsprüfer der Regierung behaupteten, den Missbrauch von Haushaltsmitteln im Verteidigungsministerium aufgedeckt zu haben. In beiden Fällen war jedoch klar, dass die Vorwürfe politisch motiviert waren. Hryzenko verteidigte sich energisch vor dem Parlament und behielt vorläufig sein Amt, Luzenko jedoch wurde für ineffektive Arbeit gerügt und am 30. November mit den Stimmen der Partei der Regionen und der Kommunisten sowie von 18 Abgeordneten des Blocks Timoschenko entlassen.

Zwei Tage später wurde Tarasjuk von der Antikrisenkoalition entlassen, obwohl seine Ernennung laut Verfassung zur Quote des Präsidenten gehörte. Mit der Unterstützung des Präsidenten und einer Gerichtsentscheidung, die die Entlassung für illegal erklärte, im Rücken weigerte sich Tarasjuk, sein Amt zu verlassen. Janukowitsch hinderte ihn jedoch daran, an Ministertreffen teilzunehmen und stoppte 
die Finanzierung des Ministeriums, was schließlich Tarasjuk dazu zwang, am 30. Januar zurückzutreten. Der erfolgreiche Ausschluss von Tarasjuk und Luzenko aus der Regierung beraubte Juschtschenko seiner Hauptverbündeten in der Regierung und stellte eine bedeutende Niederlagen im Machtkampf mit Janukowitsch dar. Die Regierungskoalition konnte Luzenko mit Hilfe von Stimmen aus dem Block Timoschenko entlassen, was die internen Widersprüche innerhalb der Opposition offenbarte. Der erzwungene Abgang von Tarasjuk war jedoch sehr viel schwerwiegender, da er das verfassungsmäßige Recht des Präsident, den Außenminister zu ernennen, in beträchtlichem Maße untergrub.

\section{Verfassungslücken}

Z s blieb weiterhin unklar, was geschehen würde, Cwenn das Parlament dem Kandidaten des Präsidenten für das Amt des Außenministers die Zustimmung verweigern würde. Dies war nur ein Beispiel für die vielen Lücken in der neuen Verfassung, die eine Klärung der Prozeduren durch das Gesetz „Über das Ministerkabinett" erforderte. Die Annahme dieses Gesetzes wurde zu einem entscheidenden Faktor im Machtkampf zwischen dem Präsidenten und der Regierungskoalition und wurde als eines der Hauptinstrumente angesehen, um die konstitutionellen Rechte der jeweiligen Seite zu verbessern.

Gleichzeitig herrscht nach wie vor Unsicherheit über die Gesetzesmäßigkeit der „Politreform“ an sich. Juschtschenko suchte weiterhin nach Mitteln, um sie zu revidieren, während die Regierungskoalition als eine ihrer ersten Maßnahmen ein Gesetz von äußerst zweifelhafter Legalitätannahm, das dem Verfassungsgericht ein Urteil über die Verfassungsmäßigkeit der Verfassungsänderungen vom Dezember 2004 verbot. Daher versuchten sowohl die Regierung als auch der Präsident, ihre konstitutionellen Rechte auf Kosten des anderen zu erweitern, indem jede Seite ihren eigenen Gesetzesvorschlag „Über das Ministerkabinett“ formulierte, um Vorteile aus den weißen Flecken in der existierenden Verfassung zu ziehen.

Beide Gesetzesvorschläge wurden ausdrücklich auf die gegenwärtigen Amtsinhaber und Umstände zugeschnitten, mit dem Ziel, ihnen einen Vorteil im Machtkampf zu verschaffen. Viele Teilnehmer und Beobachter sagten einen Kompromiss vorher, bei dem zwar der Regierungsvorschlag die Grundlage des neuen Gesetzes darstellen würde, aber einige Vorschläge des Präsidenten einbezogen würden, um sicherzustellen, dass er das Gesetz unterschriebe. Die Regierung war sich aber sicher, eine Reihe von Optionen zu haben, um ihre Präferenzen so weit wie möglich durch- zusetzen: die nötigen 300 Abgeordnetenstimmen im Parlament, um das präsidiale Veto zu überstimmen, den Einsatz der neuen Bestimmungen der Verfassung, die die Notwendigkeit einer Unterschrift unter das Gesetz durch den Präsidenten umgehen, indem der Parlamentspräsident diese leisten kann sowie ansonsten ein für die Koalition positives Urteil des Verfassungsgerichts.

Demzufolge nahm die Koalition Ende Dezember 2006 die Regierungsversion des Gesetzes an, ohne einen einzigen von Juschtschenkos Vorschlägen zu berücksichtigen. Dies hatte Juschtschenkos Veto zufolge, was wiederum am 12. Januar 2007 unter skandalträchtigen Umständen überstimmt wurde: Die erforderlichen zusätzlichen Stimmen kamen vom Block Timoschenko, der im Austausch dafür die Unterstützung der Koalition für das Gesetz über das „imperative Mandat“ für Abgeordnete von Lokalräten und das Gesetz über die Opposition (in erster Lesung) erhielt. Beide Gesetze sind für den Block Timoschenko von großer Bedeutung. Das imperative Mandat, d.h. der Verlust des Sitzes im Lokalrat beim Verlassen der Partei, auf deren Liste sie gewählt worden sind, soll den massiven Mitglieder- und Abgeordnetenschwund auf der lokalen Ebene stoppen. Das Gesetz über die Opposition soll dem Block als größter Oppositionspartei neu Gestaltungsmöglichkeiten im Parlament geben.

Timoschenkos zynisches Geschäft mit der Partei der Regionen versetzte Juschtschenkos Position einen ziemlichen Schlag, kann aber auch dazu führen, dass Timoschenko in großem Maße an Glaubwürdigkeit verliert. Daraufhin fällte der Präsident die gesetzlich fragwürdige Entscheidung, das Gesetz ein zweites Mal an das Parlament zurück zu verweisen. Nach einigem Zögern beschloss die Koalition, das Gesetz mit der Unterschrift des Parlamentspräsidenten Oleksandr Moroz in Kraft treten zu lassen, was durch die Verfassungsänderungen gestattet wurde. Das Gesetz wurde offiziell am 2. Februar 2007 veröffentlicht und war somit das erste Gesetz, das ohne die Unterschrift des Präsidenten in Kraft trat. Juschtschenko focht das Gesetz vor dem Verfassungsgericht an, um diesen ist es aber seit seiner Neukonstituierung im August ziemlich still gewesen, und er wird sich, mit seiner mangelnden Erfahrung, kaum in den Streit zwischen Präsident und Parlament einmischen.

Das Inkrafttreten des Gesetzes stellt einen überraschenden Präzedenzfall dar, provozierte Kommentatoren dazu, von Krieg zwischen und innerhalb der Regierungsgewalten zu sprechen und gab Timoschenkos Wunsch für vorzeitige Wahlen Auftrieb. Innerhalb weniger Tage hatte sich aber das 
Karussell der politischen Allianzen erneut gedreht, und am 5. Februar unterschrieb Timoschenko zusammen mit Unsere Ukraine eine Erklärung über koordinierte Opposition im Parlament. Diese beinhaltet auch die Verpflichtung, gemeinsam für die Revidierung der Politreform zu arbeiten, während die Regierungskoalition schon eine Änderung des Gesetzes diskutierte, um einige der Vorschläge des Präsidenten einzubeziehen.

\section{Resümee}

ie unklare konstitutionelle und politische Lage seit der Verabschiedung der Politreform hat dazu geführt, dass politische Akteure kurzfristige Taktiken benutzen, um Macht zu erlangen und vor allem, um ihr politisches Überleben zu sichern. Alle Akteure versuchten so, mit den Regeln zu spielen, um ihre eigene Position im zukünftigen politischen Spiel zu verbessern, statt sich an die Regeln zu halten. Dies erzeugte Bündnisse, die sich schnell änderten und auf situationsbedingt wechselnden gemeinsamen Interessen basierten. Unter den gegebenen Umständen ist dieses
Verhalten rational, bringt aber auch die Kluft zwischen Politikern und den Interessen ihrer Wähler zum Ausdruck, da Politiker sich anscheinend problemlos mit den erklärten Feinden von Gestern verbündeten.

Solche Aktionen sind auch dazu geeignet, politische und institutionelle Unsicherheit neu entstehen zu lassen. Wie Robinson (2000: 4-7) in Hinblick auf Jelzins Russland anmerkte, ist die Krux des Problems die Schwäche formaler staatlicher Regeln, die instabil und Veränderungen gemäß den Launen von Politikern unterworfen sind. Diese Schwäche (re-)produziert Instabilität, da es den Regeln nach Ansicht der rivalisierenden politischen Eliten an Legitimität mangelt. Diese Eliten haben somit wenig Anreize, sich an formale Regeln zu halten, da sie nicht sicher sein können, dass ihre Rivalen sich auch an diese Regeln halten werden und dass diese Regeln auch in Zukunft wichtig sein wird. Unter solchen Umständen ist daher Unsicherheit das einzig Sichere.

Übersetzung aus dem Englischen: Matthias Neumann

Über die Autorin

Sarah Whitmore ist Senior Lecturer in Politics an der Oxford Brookes Universität, Oxford, Großbritannien.

Im Text zitierte Literatur:

Neil Robinson (2000), 'Introduction: Institutions and Political Change in Russia', in Neil Robinson (Hg.), Institutions and Political Change in Russia, Basingstoke: Macmillan, S. 1-10.

\section{Dokumentation}

\section{Zentrale Bestimmungen des neuen Gesetzes „Über das Ministerkabinett“}

- Wenn der Präsident den Kandidaten für das Ministerpräsidentenamt, dervon der Koalition der Parlamentsfraktionen bestimmt wird, innerhalb von 15 Tagen nicht akzeptiert, kann die Koalition den Ministerpräsidenten ohne Zustimmung des Präsidenten ernennen.

- Der Präsident ernennt den Verteidigungs- und den Außenminister nur auf den Vorschlag der Regierungskoalition hin. Wenn der Präsident sich weigert, Kandidaten vorzuschlagen, werden diese Minister vom Parlament ernannt.

- Die Regierung verwaltet das Staatseigentum. Dies bedeutet, dass der Fond für Staatsvermögen, der unter der Kontrolle des Präsidenten stand, in die Kompetenz der Regierung überführt werden sollte.

- Das Ministerkabinett wird als legitimiert angesehen, sobald zwei Drittel seiner Mitglieder den Amtseid abgelegt haben.

- Präsidialerlasse müssen vom Ministerpräsidenten und dem Minister des jeweiligen Geschäftsbereiches gegengezeichnet werden und können mit Kommentaren an den Präsidenten zurückverwiesen werden.

Quelle: Ukrainska Prawda, www.pravda.com.ua., 15. Januar 2007 


\section{Analyse}

\section{Ukrainisch - Russisch: Sprachen, Sprachgebrauch, Sprachenkonflikte in der Ukraine}

Gerhard Simon, Universität zu Köln

\section{Zusammenfassung}

Die Volkszählung von 2001 zeigt einen Anstieg des ukrainischen und entsprechend einen Rückgang des russischen und russischsprachigen Bevölkerungsanteils seit 1989. Verbreitung und Ansehen der ukrainischen Sprache haben zugenommen. Dennoch hat das Russische im Osten und Süden des Landes im öffentlichen Raum eine beherrschende Stellung behalten. Besonders weitgehend ist die Ukrainisierung des Bildungswesens fortgeschritten. In den Massenmedien und der Popkultur überwiegt dagegen das Russische. In den vergangenen 15 Jahren ist es nicht zu Dauerkonflikten in der Sprachenfrage gekommen, aber es gibt ein latentes Konfliktpotenzial. Zwar reagiert die Bevölkerung in weiten Teilen des Landes gelassen im Sprachenstreit, aber insbesondere im Osten und Süden sind die postsowjetischen Eliten in Krisensituationen in der Lage, die Sprachenfrage für die eigenen politischen Belange zu instrumentalisieren.

\section{Einleitung}

$\mathrm{D}$ ie Ukraine ist ein zweisprachiges Land, viele, aber durchaus nicht alle Menschen beherrschen sowohl die ukrainische als auch die russische Sprache. Der Sprachgebrauch ist innerhalb des Landes außerordentlich unterschiedlich; im Osten und Süden hat das Russische, im Westen das Ukrainische eine beherrschende Stellung. In der Zentralukraine mit Kiew sind beide Sprachen etwa gleich präsent, wobei hinsichtlich der funktionalen Ebenen im Sprachgebrauch vielfache Unterschiede bestehen. Eindeutige Grenzen gibt es weder territorial noch funktional. Neben dem Ukrainischen und Russischen haben andere Sprachen im öffentlichen Raum nur lokal begrenzte Bedeutung, wie das Rumänische in der Bukowina oder das Krimtatarische auf der Krim.

\section{Historischer Hintergrund}

乙istorische Entwicklungen und Sprachenpolitik haben seit dem 19. Jahrhundert immer wieder zu massiven Verschiebungen im Sprachgebrauch geführt. In jenen Teilen der Ukraine, die bis 1918 zum Russischen Reich gehörten, versuchte die zarische Regierung mit großem Nachdruck das Russische im öffentlichen Raum durchzusetzen. Das Jahrzehnt nach dem Sieg der Bolschewiki im Bürgerkrieg war geprägt durch die Umkehrung dieser Politik: In der Zeit der Korenizacija (Einwurzelung) förderten die Bolschewiki das Ukrainische im öffentlichen Raum und drängten das Russische zurück. Seit den frühen 1930er Jahren erlahmte diese Politik der Ukrainisierung und verkehrte sich immer mehr in ihr Gegenteil bis zur aggressiven Sprachenpolitik in der Breshnew-Zeit, die das Ziel verfolgte, das Russische im Bildungswesen nach und nach als einzige Unterrichtssprache durchzusetzen.

Das erwies sich in der Westukraine, die erst im Zuge des Zweiten Weltkriegs mit der Sowjetukraine vereinigt worden war, als ausgeschlossen. Die Westukraine war nach der Vernichtung der jüdischen Bevölkerung während des Holocaust und nach der Aussiedlung der Polen weitgehend ethnisch und sprachlich homogen ukrainisch. Dagegen zogen mit der fortschreitenden Industrialisierung seit den 1930er Jahren Hunderttausende von Russen in die östlichen und zentralen Regionen der Ukraine und verstärkten die ohnehin vorhandene russische sprachliche und kulturelle Prägung, vor allem der Städte. Die jahrhundertelange Zugehörigkeit zum Russischen Reich, die massive Zuwanderung von Russen in sowjetischer Zeit und die russifizierende Sprachenpolitik in spätsowjetischer Zeit haben dazu geführt, dass im Osten, Süden und in der Zentralukraine für Millionen von ethnischen Ukrainern das Russische die Muttersprache ist. Bei der Volkszählung im Dezember 2001 gaben von den 37,54 Millionen Ukrainern 5,54 Millionen Russisch als ihre Muttersprache an.

Obwohl nur 17,3\% der Bevölkerung ethnische Russen sind, erklären so gemäß dem soziologischen Monitoring des Instituts für Soziologie der Nationalen Akademie der Wissenschaften der Ukraine 34\% der ukrainischen Bevölkerung, Russisch sei ihre Muttersprache. 64\% bezeichneten Ukrainisch als ihre Muttersprache. (Siehe Grafiken 1 und 2 auf Seite 12) Wir haben also davon auszugehen, dass knapp zwei Drittel der Bevölkerung ukrainophon, etwas mehr als ein Drittel russophon sind. Dabei war der Anteil der Russophonen im vergangenen Jahrzehnt leicht rückläufig, der Anteil der Ukrainophonen wuchs geringfügig, wie die Grafiken 3 und 4 zeigen.

Dies ist eine Folge der Sprachenpolitik in der seit 1991 unabhängigen Ukraine. Noch vor der Ausrufung der Unabhängigkeit erklärte das Sprachengesetz 


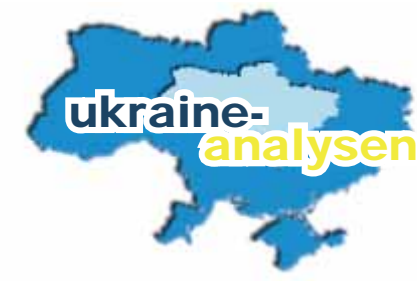

vom 28. Oktober 1989 Ukrainisch zur einzigen Staatssprache auf dem Territorium der zunächst noch zur Sowjetunion gehörenden Ukraine und verkehrte damit die sowjetische Sprachenpolitik der zurückliegenden Jahrzehnte ins Gegenteil. Die Zurückweisung der russifizierenden Sprachenpolitik durch die neuen nationalen postkommunistischen Eliten in vielen Unionsrepubliken und nachmaligen neuen unabhängigen Staaten hat bei der Demontage der Sowjetunion eine wichtige Rolle gespielt, weil hierin ein erhebliches Mobilisierungspotenzial für die Unabhängigkeit und gegen den sowjetischen Zentralismus steckte.

\section{Nationalität und Sprachkompetenz}

$\mathrm{D}$ ie Ergebnisse der Volkszählung vom Dezember 2001 dokumentieren die nationale Zusammensetzung der Bevölkerung und die Sprachensituation zehn Jahre nach der Erklärung der Unabhängigkeit. Sie erlauben auch Vergleiche mit der letzten Volkszählung in sowjetischer Zeit, die 1989 stattfand. Die Gesamtbevölkerung des Landes verminderte sich von 51,5 Millionen auf 48,5 Millionen. In der nationalen Zusammensetzung ergaben sich deutliche Verschiebungen zugunsten der Ukrainer, deren Anteil an der Bevölkerung von 73\% auf 78\% anstieg; der Anteil der ethnischen Russen fiel entsprechend von 22\% 1989 auf 17\% im Jahr 2001. Dies dürfte teilweise durch ein negatives Migrationssaldo, zum Teil aber auch dadurch zu erklären sein, dass manche Personen mit einer labilen ethnischen Identität sich jeweils für die Ethnie entschieden, die das höhere Prestige besaß. 1989 waren das die Russen und 2001 die Ukrainer. Schließlich muss bei Volkszählungen weder die ethnische $\mathrm{Zu}$ gehörigkeit noch die Sprachkompetenz nachgewiesen werden. Registriert wird die Selbsteinschätzung. Wie schon 1989 stellen auch 2001 unter allen ukrainischen Regionen lediglich auf der Krim die Russen die Bevölkerungsmehrheit. Die am stärksten ethnisch russisch geprägte Großstadt ist Sewastopol auf der Krim, wo 2001 der russische Bevölkerungsanteil 72\% gegenüber einem ukrainischen Anteil von 22\% betrug.

Es war schon davon die Rede, dass in der Ukraine ethnische Zugehörigkeit und Sprachverhalten in vielen Teilen des Landes weit auseinander klaffen. 85\% der Ukrainer sagten, Ukrainisch sei ihre Muttersprache, $15 \%$ nannten das Russische als ihre Muttersprache. Bei den Russen gaben dagegen 96\% die Sprache ihrer Ethnie als Muttersprache an, 4\% das Ukrainische. Außer den Russen sind auch zahlreiche andere ethnische Minderheiten in der Ukraine überwiegend russophon, so z. B. $63 \%$ der 276.000 Belorussen, $83 \%$ der 104.000 Juden, $89 \%$ der 92.000 Griechen und 65\% der 33.000 Deutschen. Dies ist das Erbe des
Imperiums, als sozialer Aufstieg und Assimilation auf dem Territorium der Ukraine über die russische, keineswegs über die ukrainische Sprache führten.

Die Ukrainer mit russischer Muttersprache leben ganz überwiegend im Osten und Süden des Landes, im Westen ist ihre Zahl verschwindend gering. So bezeichneten 59\% der Ukrainer im Gebiet Donezk, 26\% im Gebiet Charkiw, 28\% im Gebiet Odesa, aber nur $0,4 \%$ im Gebiet Lemberg das Russische als ihre Muttersprache.

Neben der Muttersprache wurde 2001 ebenso wie früher bei den sowjetischen Volkszählungen nach der Beherrschung anderer Sprachen gefragt. Auf diese Weise gibt die Volkszählung Auskunft darüber, wie viele Russen in der Ukraine das Ukrainische beherrschen und wie viele Ukrainer das Russische. Von den 8,3 Millionen Russen gaben 55\% an, das Ukrainische frei zu beherrschen. Umgekehrt sagten von den 37,5 Millionen Ukrainern 43\%, sie könnten sich frei auf Russisch ausdrücken. Addiert man also bei den Ukrainern die Menschen mit russischer Muttersprache und diejenigen, die frei die russische Sprache beherrschen, zeigt sich, dass 58\% der ethnisch ukrainischen Bevölkerung ohne Schwierigkeiten in russischer Sprache kommunizieren können. $\mathrm{Zu}$ einem ähnlichen Ergebnis kommt man bei den Russen hinsichtlich ihrer Sprachkompetenz im Ukrainischen: 59\% der ethnischen Russen im Land können sich frei auf Ukrainisch ausdrücken.

Dies erscheint auf den ersten Blick und gewiss im internationalen Vergleich als ein hoher Grad an Zweisprachigkeit. Aber es bedeutet auch, dass mehr als $40 \%$ der ethnischen Russen und Ukrainer die Sprache des anderen nicht frei und ungezwungen verwenden können. Daraus folgt gewiss keine totale Sprachlosigkeit miteinander, denn neben der freien Beherrschung einer anderen Sprache gibt es viele Abstufungen der Kommunikationsmöglichkeit. Aber die manchmal geäußerte Behauptung, es gebe eigentlich kein Konfliktpotential in der Sprachenfrage, denn alle könnten miteinander frei kommunizieren, trifft nicht zu.

Wie nicht anders zu erwarten, bestehen auch hinsichtlich der Beherrschung der Sprache des anderen gravierende regionale Unterschiede. Im Gebiet Donezk beherrschen von den 1,8 Millionen Russen 55\% das Ukrainische, mit anderen Worten, fast die Hälfte der russischen Bevölkerung gibt an, nicht frei auf ukrainisch kommunizieren zu können. Umgekehrt sagen von den 2,7 Millionen Ukrainern im Gebiet Donezk 90\% Russisch sei entweder ihre Muttersprache (59\%) oder sie beherrschten es frei (31\%). Daraus ergibt sich ein durchgehend russisch- 


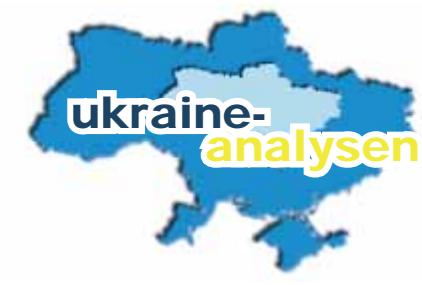

sprachiger Kommunikationsraum. Spiegelbildlich stellt sich die Sprachensituation im Gebiet Lemberg dar. Von den 2,5 Millionen Ukrainern beherrschen $18 \%$ das Russische, mit anderen Worten über $80 \%$ können sich nicht frei in der russischen Sprache bewegen. Von den 93000 Russen im Gebiet Lemberg sagen $89 \%$ das Ukrainische sei entweder ihre Muttersprache $(12 \%)$ oder sie beherrschten die ukrainische Sprache (77\%). Es zeigt sich, dass im Gebiet Lemberg ein durchgehend ukrainischer Kommunikationsraum besteht.

Eine recht ausgeprägte Zweisprachigkeit und damit eine Mittelposition weist die Volkszählung für die Stadt Kiew aus. Von den 2,1 Millionen Ukrainern beherrschen $58 \%$ das Russische, für weitere $14 \%$ ist es die Muttersprache, so dass $72 \%$ der ukrainischen Bewohner von Kiew angeben, auf Russisch frei zu kommunizieren. Von den 337000 Russen in der Stadt können $71 \%$ in ukrainischer Sprache kommunizieren, für $8 \%$ ist das Ukrainische die Muttersprache, so dass insgesamt $79 \%$ der russischen Bevölkerung des Ukrainischen mächtig sind.

Die Wirklichkeit ist noch weit komplizierter als diese Zahlen ohnehin vermuten lassen, denn weder die Sprachen noch der Sprachgebrauch sind so klar voneinander abgegrenzt wie die Volkszählungsdaten auf den ersten Blick nahe legen. Außerdem sind zahlreiche Mischformen des Ukrainischen und Russischen entstanden, die als Surshik bezeichnet werden, des weiteren bestehen erhebliche dialektische Unterschiede innerhalb des Ukrainischen zwischen den verschiedenen Landesteilen. Vor allem aber gibt es eine große Zahl von Menschen, die sich weder ethnisch noch hinsichtlich des Sprachgebrauchs eindeutig dem Ukrainischen oder Russischen zuordnen lassen oder zugeordnet werden wollen.

Einen Eindruck davon vermitteln die Antworten auf die Frage: „Welche Sprache sprechen Sie normalerweise zu Hause?" (siehe auch Grafik 3 auf Seite 12). $29 \%$ der in der ganzen Ukraine repräsentativ Befragten gaben 1994 an, Ukrainisch und Russisch nebeneinander zu gebrauchen. Dieser Anteil fiel auf 22\% im Jahr 2005. Im Jahr nach der Orangen Revolution sagten $42 \%$ der Menschen, das Ukrainische sei gewöhnlich ihre Umgangssprache zu Hause, für 36\% war es das Russische. Interessanterweise ist in beiden Fällen dieser Anteil im zurückliegenden Jahrzehnt zu Lasten des gleichzeitigen Sprachgebrauchs gestiegen. Ein Vergleich mit den Angaben zur Muttersprache (siehe Grafik 4 auf Seite 13) legt nahe, dass die Russophonen stärker an ihrer Sprache festhalten als die Ukrainophonen. Denn der Anteil der russischen Muttersprachler entspricht weitgehend dem Anteil derjenigen, die angeben, zu Hause überwiegend Russisch zu sprechen, während ein erheblicher Teil (mehr als 20\%) der ukrainischen Muttersprachler im Alltag auch das Russische gebraucht. Vielleicht ist es ein Erbe der imperialen Vergangenheit, dass Ukrainischsprecher im Alltag viel eher bereit sind zum Russischen zu wechseln als umgekehrt. Auch statistische Untersuchungen unter Schülern und Studenten in Kiew bestätigen, dass die Bereitschaft zum Sprachwechsel bei Personen mit ukrainischer Muttersprache weit größer ist als bei Jugendlichen mit russischer Muttersprache.

\section{Sprachengesetz und Sprachenprestige}

as Sprachengesetz vom 28. Oktober 1989, das auf dem Höhepunkt der Perestroika in der Sowjetunion das Ukrainische zur einzigen Staatssprache auf dem Territorium der Ukrainischen SSR erklärte, gilt bis heute mit geringfügigen Änderungen aus dem Jahr 1995 fort. Die Verfassung von 1996 bestätigte in Artikel 10 die Bestimmungen über die Staatssprache. Dabei schließt das Sprachengesetz keinesfalls das Russische aus dem öffentlichen Leben aus. Es legt vielmehr fest, dass in staatlichen Organen einschließlich des Justizwesens, in Organisationen, im Bildungswesen und in der Wirtschaft neben dem Ukrainischen auch das Russische („die Sprachen der anderen Nationalitäten“ in der Formulierung des Gesetzes) Verwendung findet in Territorien, in denen die Russen die Bevölkerungsmehrheit bilden.

Seit 1994 haben einige Gebiete (Donezk, Luhansk) und Großstädte des Ostens und Südens (u.a. Odesa, Mykolajiw) das Russische faktisch als „offizielle Sprache“ oder „zweite Staatssprache“ eingeführt, obgleich dies dem Sprachengesetz widerspricht. In der Autonomen Republik Krim galten bis zur neuen Krimverfassung 1998 das Russische, Ukrainische und Krimtatarische als Staatssprachen. Der amtliche Schriftverkehr innerhalb der Regionen erfolgt in der Zentral- und Westukraine sowie mit Kiew als Landeshauptstadt auf Ukrainisch, im Osten und Süden des Landes auf Russisch. Beamte sind laut Sprachengesetz verpflichtet, beide Sprachen zu kennen. Diese Vorschrift ist jedoch wie die meisten anderen Sprachenbestimmungen nicht mit Kontrollen und Sanktionen bewehrt. Die Nichteinhaltung der Sprachenvorschriften wird nicht mit Strafen bedroht. Damit sind dem Staat bei der Ukrainisierung enge Grenzen gesetzt. Hierin unterscheidet sich die Sprachenpolitik in der Ukraine deutlich von der postkommunistischen Sprachenpolitik in Estland oder Lettland, aber auch von der Ukrainisierung in den 1920er Jahren, als die Sowjetorgane auch mit rigorosen Disziplinarmaßnahmen das Russische in der Ukraine zurückdrängten. 


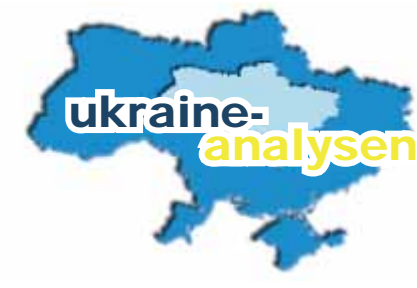

Das Russische ist also im öffentlichen Raum präsent und hat im Osten und Süden eine beherrschende Stellung. Insoweit kann von einer Diskriminierung der russischen Sprache, wie vielfach von russischer Seite in der Ukraine und in Russland behauptet und beklagt wird, keine Rede sein. Andererseits haben sich seit sowjetischer Zeit tief greifende Veränderungen vollzogen: Ansehen und Verbreitung der ukrainischen Sprache haben deutlich zugenommen und nehmen weiter zu. Während in sowjetischer Zeit eine Karriere und sozialer Aufstieg ohne gute Russischkenntnisse ausgeschlossen waren, gilt jetzt das Umgekehrte. Ohne die Beherrschung der ukrainischen Sprache kann man - abgesehen von einigen Gebieten im Osten und auf der Krim - keine Karriere mehr machen. Während in sowjetischer Zeit das Erfordernis der Bilingualität (Muttersprache und Russisch) für die Ukrainer galt, besteht diese Forderung also heute für die ethnischen Russen und russophonen Ukrainer. Diese Umkehr des Erfordernisses der Bilingualität, das jetzt an die Russen statt wie früher an die Ukrainer gestellt wird, erscheint einem Teil der Betroffenen als Diskriminierung.

Die Sprache ist wie stets ein wesentlicher Marker der nationalen Identität. Deshalb kann der neue ukrainische Staat nicht auf Sprachenpolitik und die Förderung der ukrainischen Sprache verzichten. Die Sprachenpolitik war insgesamt ausgesprochen gemäßigt. Dies wird auch dadurch bestätigt, dass sowohl die Lobbyisten des Russischen als auch die einer rascheren Ukrainisierung heftige Kritik an ihr üben.

Muss manukrainisch sprechen, um ein ukrainischer Patriot zu sein? Keineswegs. Auch viele russisch sprechende Ukrainer und ethnische Russen identifizieren sich zunehmend mit dem neuen Staat und sehen sich als dessen Bürger. Es gibt in der Ukraine keinen prorussischen Separatismus, allerdings existiert im Osten und Süden eine Sowjetnostalgie. Das hat sich während der Orangen Revolution gezeigt, als autonomistische und potenziell separatistische Aktionen nach wenigen Tagen zusammenbrachen. Auf dem Majdan wurde zwar überwiegend von der Rednertribüne her ukrainisch gesprochen, aber Juschtschenko hat sich demonstrativ auch in russischer Sprache an die Menge gewandt. Auseinandersetzungen und Konflikte um die Sprache werden dadurch gemildert, dass eine Gleichsetzung von Sprachgebrauch und nationaler Identifizierung an der Wirklichkeit vorbei geht.

\section{Die Ukrainisierung des Bildungswesens}

ie mit Abstand größten Erfolge der Ukrainisierung sind im Bildungswesen $\mathrm{zu}$ verzeichnen, wie die Tabellen 1 bis 3 auf den Seiten 14 bis 16 aus- weisen. Überall ist der Prozentanteil der Kinder, die ukrainische Kindergärten und Schulen besuchen, im vergangenen Jahrzehnt zum Teil drastisch gestiegen. Dies ist eine Investition in und für die Zukunft. Sollte das neue Gewicht der ukrainischen Sprache auf dem jetzigen Niveau für einige Jahrzehnte erhalten bleiben, würde das Ukrainische wohl die beherrschende Sprache im Land werden, ohne dass das Russische deshalb aus dem öffentlichen Raum verschwindet. Der Prozentanteil der Kinder in allgemein bildenden Schulen mit ukrainischer Unterrichtssprache entsprach 2005 mit 78\% ziemlich genau dem Anteil der Ukrainer an der Gesamtbevölkerung.

Allerdings zeigt ein Blick in die Regionen, dass wie meist die Verhältnisse höchst unterschiedlich sind. In vielen Regionen des Ostens und Südens ist das Russische als Unterrichtssprache in den Schulen nach wie vor stärker präsent als es dem Anteil der ethnischen Russen entspricht, in der Zentralukraine und im Westen sind die Verhältnisse umgekehrt. Auf der Krim besuchen nur 5\% der Schüler ukrainische Schulen, obwohl der Anteil der Ukrainer an der Bevölkerung fast fünfmal so groß ist. Für das Gebiet Donezk lauten die entsprechenden Zahlen 29\% und $38 \%$. Umgekehrt, im Gebiet und in der Stadt Kiew mit 6\% bzw. 13\% Russen besuchen nur 1\% bzw. 4\% der Schüler russischsprachige Schulen. So wirkt das allgemein bildenden Schulwesen in manchen Teilen des Ostens und Südens nach wie vor russifizierend, im Zentrum und im Westen dagegen ukrainisierend. In manchen Gebieten zwischen dem Zentrum und dem Osten entspricht die Zahl der Schüler in ukrainischen und russischen Schulen in etwa dem jeweiligen Anteil an der Bevölkerung, so in den Gebieten Mykolajiw, Charkiw und Dnipropetrowsk. Während das Ukrainische in den Schulen mit russischer Unterrichtssprache Pflichtfach ist, gilt das umgekehrt nicht. Deshalb gibt es im Westen viele Schulen, in denen Russisch gar nicht oder auf einem recht niedrigen Niveau unterrichtet wird. Grafik 6 auf Seite 17 macht deutlich, dass drei Viertel der Menschen in der Ukraine Russischunterricht an den Sekundarschulen wünschen, allerdings ist der Wunsch nach Englischunterricht noch populärer.

Noch stärker als im allgemein bildenden Schulwesen hat sich die ukrainische Sprache im vergangenen Jahrzehnt in den Kindergärten und an den Hochschulen durchgesetzt. 82\% der Studenten studierten 2005 in ukrainischer Sprache und 84\% der Kinder besuchten ukrainischsprachige Kindergärten, damit liegen diese Zahlen leicht über dem Bevölkerungsanteil der Ukrainer. Die für das Schulwesen gemachten grundsätzlichen Beobachtungen gelten auch für die 


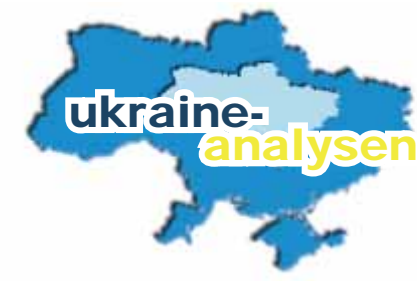

Hochschulen: Im Osten und Süden (Krim, Donezk) ist der Anteil der in Russisch Studierenden größer als der russische Bevölkerungsanteil; im Zentrum (Gebiet und Stadt Kiew) ist dagegen der Anteil der Studierenden mit ukrainischer Studiensprache größer als der ukrainische Bevölkerungsanteil. In manchen Gebieten dazwischen (Dnipropetrowsk, Charkiw) entsprechen die Zahlen einander.

Bei den Kindergärten ist in der Regel der Anteil der Kinder, die ukrainischsprachige Einrichtungen besuchen, größer als der Anteil der Ukrainer an der Bevölkerung des Gebietes. Mit anderen Worten, die Vorschuleinrichtungen wirken ukrainisierend. Ausnahmen sind die Krim und das Gebiet Luhansk. In der gesamten westlichen Ukraine, aber auch in Stadt und Gebiet Kiew gibt es faktisch keine russischsprachigen Kindergärten mehr.

\section{Die Massenmedien}

Tn deutlichem Kontrast zum Bildungswesen hat das 1 Russische nach wie vor eine starke, teilweise beherrschende Stellung in den Massenmedien und in der Pop- und Massenkultur. Dafür gibt es vor allem zwei Gründe:

1. Die Einwirkungsmöglichkeiten des Staates in diesen Bereichen sind begrenzt, weil vielfach privates Kapital nach Marktgesichtspunkten entscheidet. Weil russische Bücher, Zeitungen und Fernsehprogramme auch von Ukrainophonen gelesen und gesehen werden, ist der Markt dafür größer als für Produkte in ukrainischer Sprache, die von Russophonen eher zögernd angenommen werden.

2. Sowohl gedruckte wie auch elektronische Produkte werden preisgünstig aus Russland eingeführt. Das gilt insbesondere für den Buch- und Filmmarkt und hier vor allem für Unterhaltungsliteratur, von der Millionen Exemplare auf dem ukrainischen Markt vertrieben werden.

Übersetzungen aus Fremdsprachen ins Ukrainische verursachen erheblich mehr Kosten pro Exemplar als ins Russische, weil der russischsprachige Markt eben viel größer ist. Die ca. 800 privaten Verlage in der Ukraine publizieren überwiegend russischsprachige Produkte. Auch bei Zeitschriften und Zeitungen hat das Russische eine führende Position. Nur 23\% der verkauften Zeitschriftenexemplare und 27\% der verkauften Zeitungen erschienen im Jahr 2000 in ukrainischer Sprache. Zwar stieg die Zahl der ukrainischen Zeitungstitel seit 1990, aber zugleich sank ihr Auflagenanteil im Vergleich zu den russischsprachigen Zeitungen. Auch der weit überwiegende Teil der ausländischen Filme, die in ukrainischen Kinos gezeigt werden, läuft in russischer Synchronisation. Die
Produktionen aus Hollywood machen etwa 90\% der in der Ukraine gezeigten Kinofilme aus.

Auch im Radio und Fernsehen dominiert das Russische nach wie vor. Nach einer Untersuchung der OSZE im Jahr 2000 wurden fast zwei Drittel der gesamten TV- und Radiosendezeit in russischer Sprache ausgestrahlt. Dabei überwog das Ukrainische in den staatlichen und das Russische in den privaten Sendeanstalten.

\section{Konflikte und Konfliktpotenzial}

Konflikte um Sprachen bergen stets ein erhebliches LMobilisierungspotenzial in sich, weil im Prinzip jedermann davon betroffen ist. Umso bemerkenswerter ist es, dass sich die Auseinandersetzungen in der Ukraine in den vergangenen 15 Jahren insgesamt in Grenzen gehalten haben. Zu Gewalttätigkeiten ist es nicht gekommen. Der wichtigste Grund dafür dürfte sein, dass die Ukrainer die Distanz zwischen sich und den Russen als gering einschätzen. Das soziologische Monitoring durch das Kiewer Institut für Soziologie zeigt für den Zeitraum 1994-2005 durchgängig die geringste Distanz der Ukrainer zu den Russen (nach der Bogardus Skala) im Vergleich zu zahlreichen Völkern Osteuropas und der Welt. Das entschärft den Sprachenstreit, der offenbar als ein Streit zwischen zwei Sprachen wahrgenommen wird, die im Prinzip beide ein autochthones Lebensrecht im Land haben.

Der überwiegende Teil der Menschen steht der Sprachenfrage leidenschaftslos gegenüber. Viele Zweisprachige, die ja die Bevölkerungsmehrheit bilden, sehen gar keinen Sprachenkonflikt oder spielen ihn herunter. Zur Gelassenheit trägt wohl auch die Überzeugung der Ukrainophonen bei, dass die Zeit für die ukrainische Sprache arbeitet. Eben diese Einschätzung führt auf der andern Seite immer wieder zu gereizten Reaktionen der Russophonen, die sich langfristig bedroht sehen. Insgesamt ist eine nicht überwältigende aber stabile Mehrheit für einen offiziellen Status der russischen Sprache, wie Grafik 7 auf Seite 17 zeigt.

Zwar war die Sprachenfrage in den vergangenen 15 Jahren kein Dauerthema in den innenpolitischen Auseinandersetzungen, aber in Krisenzeiten haben die politischen Eliten das Thema genutzt, um ihre Anhänger zu mobilisieren. Das geschah 1994, als die Streiks schließlich zu vorgezogenen Präsidentschaftswahlen führten, und 2004-2006 während der Orangen Revolution und der nachfolgenden anti-orangen Reaktion. Mobilisierung und Instrumentalisierung der Sprachenfrage gingen von den anti-orangen russophonen Kräften im Osten und Süden des Landes aus. Während des Präsident 
schaftswahlkampfes im Herbst 2004 wurde Viktor Juschtschenko im Osten als der Kandidat kolportiert, der die russischen Schulen und Kirchen schließen und alle zwingen würde ukrainisch zu reden. Die radikal-nationale pro-ukrainische Polemik unterstellt im Gegenzug, wer für die russische Sprache eintritt, spaltet das Land und besorgt das Geschäft Moskaus.

Insbesondere nach den Parlamentswahlen im März 2006 kam es zu einer massiven Verschärfung des Sprachenkonflikts. Einerseits schienen die Ergebnisse der Wahl anfangs eine Fortsetzung der orangen Koalition in Kiew zu ermöglichen andererseits hatte die „Partei der Regionen“ mit eindeutigen Wahlsiegen im Osten und Süden klare Mehrheiten in den dortigen Gebiets- und Stadtparlamenten errungen. Sie nutzte diese und zahlreiche Gebiets- und Stadtparlamente erklärten in den folgenden Monaten das Russische zu einer offiziellen „regionalen Sprache“, die gleichberechtigt neben dem Ukrainischen oder statt des Ukrainischen in allen öffentlichen Bereichen zur Anwendung kommen sollte. Das Stadtparlament von Charkiw war schon vor der Wahl am 6. März vorangegangen, es folgten das Gebietsparlament von Luhansk am 25. April, das Stadtparlament von Sewastopol am 26. April, das Gebietsparlament von Donezk am 18. Mai, das Stadtparlament von Dnipropetrowskam 26. Mai und das Gebietsparlament von Charkiw am 3. Juni 2006.

Der Politische Rat der „Partei der Regionen“ stellte sich in einer Erklärung „Über den Schutz der verfassungsmäßigen Rechte der russisch sprechenden Bürger der Ukraine“ vom 17. Mai 2006 ausdrücklich hinter diese Aktionen, erinnerte daran, dass die Erhebung des Russischen zur zweiten Staatssprache zum Wahlprogramm der "Partei der Regionen“ gehörte und beschuldigte die gegenwärtige Macht, d.h. Präsident Juschtschenko und die orange Regierung, die Bürgerrechte der russisch sprechenden Bürger zu verletzen.

In allen Fällen beriefen sich die gesetzgebenden Körperschaften in den Provinzen auf die „Europäische Charta der Regional- oder Minderheitensprachen“, die vom ukrainischen Parlament im Mai 2003 ratifiziert worden war. Die russische Sprache wurde damit de facto mit einer vom Aussterben bedrohten Sprache gleichgestellt, zu deren Schutz besondere staatliche Maßnahmen erforderlich sind. Das Justizministerium in Kiew zögerte nicht, auf diese groteske Verzerrung der Intention der Europäischen Charta hinzuweisen. Außerdem machte das Ministerium in seiner Stellungnahme deutlich, dass Stadt- und Gebietsparlamente keine Kompetenz haben, über den Status von Sprachen zu entscheiden. In der Sache widersprächen die Beschlüsse zudem der Verfassung und dem Sprachengesetz von 1989.

Auch zahlreiche andere Politiker des orangen Lagers stellten sich gegen die Sprachenbeschlüsse im Osten. Julia Timoschenko sprach von „verfassungswidrigen Entscheidungen“ und „Sprachenseparatismus“. Es wurden die Gerichte angerufen. Bislang ist nicht klar, ob und in welchem Umfang die Sprachenbeschlüsse der Provinzlegislativen umgesetzt wurden. Darüber hinaus ist zu bezweifeln, ob eine Umsetzung an der zuvor schon bestehenden Situation viel ändern würde. Die russische Sprache hat in den Städten des Ostens und Südens ohnehin eine starke Stellung, die nicht zuletzt durch das Sprachengesetz von 1989 geschützt wird, das die Verwendung der russischen Sprache in der lokalen und regionalen Verwaltung als Arbeitsund Amtssprache dort vorsieht, wo die Russen die Mehrheit der Bevölkerung bilden. Aber die Vorgänge im Frühsommer 2006 haben ohne Zweifel die politischen Emotionen angeheizt.

Ähnliches gilt von einer offiziellen Verlautbarung des russischen Außenministeriums vom 27. September 2006, in der die Sprachenpolitik der Stadt IwanoFrankiwsk scharf angriffen wurde. Es sei jetzt verboten, in Schulen russisch zu sprechen oder in der Öffentlichkeit russische Bekanntmachungen anzubringen. Eine „Spracheninquisition“ solle verhindern, dass Massenveranstaltungen auf Russisch durchgeführt werden. Zahlreiche Journalisten schwärmten daraufhin nach Iwano-Frankiwsk aus. Am Ende stellte der russische Konsul in Lemberg fest, die vom russischen Außenministerium verbreiteten Informationen und zahlreiche russische Presseartikel in dieser Sache seien „falsch und unbegründet“. Diese wenigen Beispiele zeigen, welche potentielle Sprengkraft in den Sprachenkonflikten steckt. Auch in Zukunft wird Gelassenheit nötig sein, um Eskalationen zu verhindern.

Über den Autoren:

Prof. Dr. Gerhard Simon ist apl. Prof. für Osteuropäische Geschichte an der Universität zu Köln und Lehrbeauftragter am Institut für Politik und Gesellschaft der Universität Bonn.

Lesetipps:

- Laada Bilaniuk: Contested Tongues. Language Politics and Cultural Correction in Ukraine, Ithaca 2005.

- Irma Oswald: Der ukrainisch-russische Sprachkontakt, in: Juliane Besters-Dilger (Hrsg.): Die Ukraine in Europa, Wien 2003, S. 309-335

- Juliane Besters-Dilger: Die aktuelle Sprachensituation in der Ukraine, in: Österreichische Osthefte 42, 4-5, 2000, S.497-523 


\section{Tabellen und Grafiken zum Text}

\section{Statistiken und Umfragen zur Sprachenfrage}

Grafik 1: Ukrainische Bevölkerung nach Nationalität

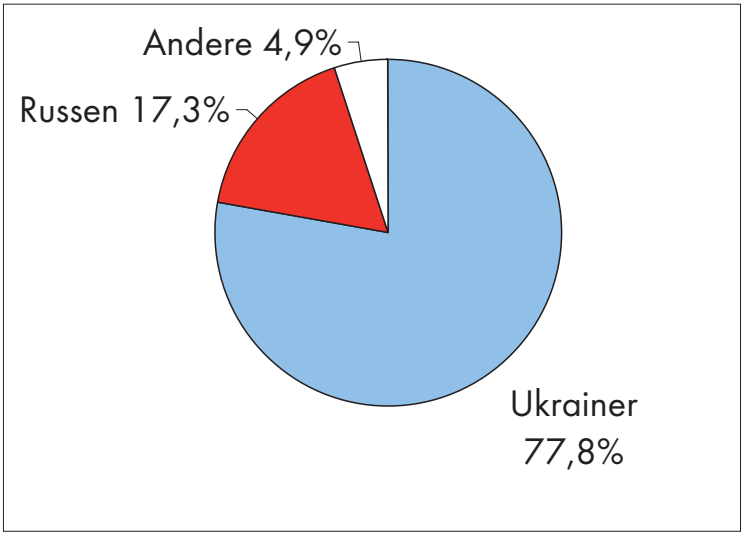

Quelle: Volkszählung 2001, http:/ / ww w.ukrcensus.gov.ua/ rus/ results/general/ nationality/
Grafik 2: Ukrainische Bevölkerung nach Muttersprache

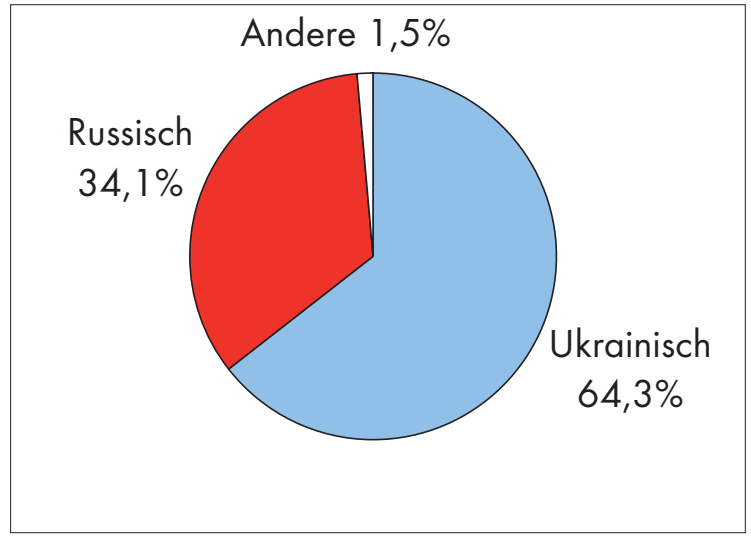

Quelle: Natalya Panina: Ukrainian Society: 1994 - 2005, Sociological Monitoring. Institute of Sociology, National Academy of Sciences of Ukraine, Kiew 2005, S. 58

Grafik 3: Welches ist Ihre Muttersprache?

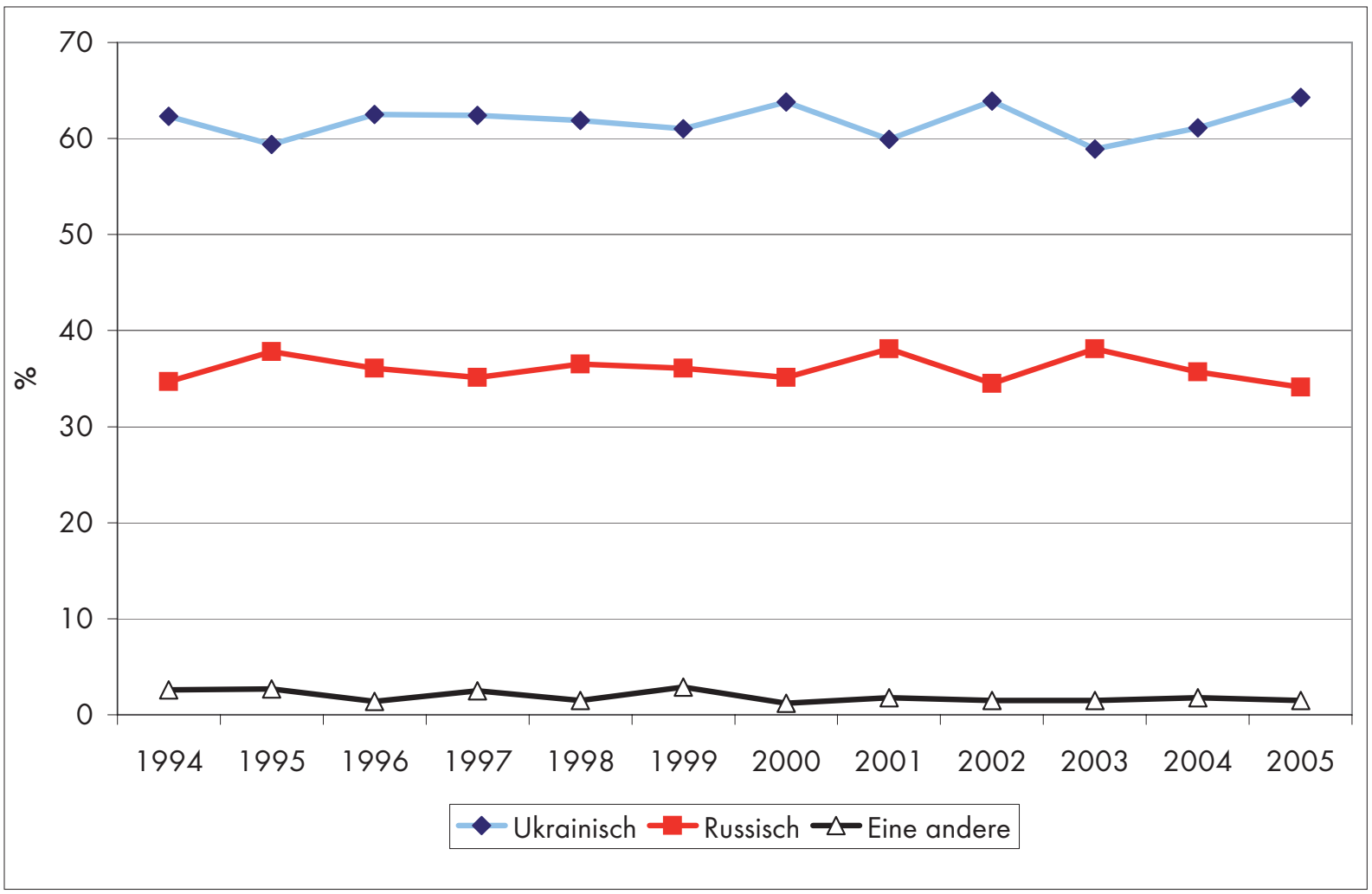

Quelle: Natalya Panina: Ukrainian Society: 1994 - 2005, Sociological Monitoring. Institute of Sociology, National Academy of Sciences of Ukraine, Kiew 2005, S. 58 
Grafik 4: Welche Sprache sprechen Sie normalerweise zu Hause?

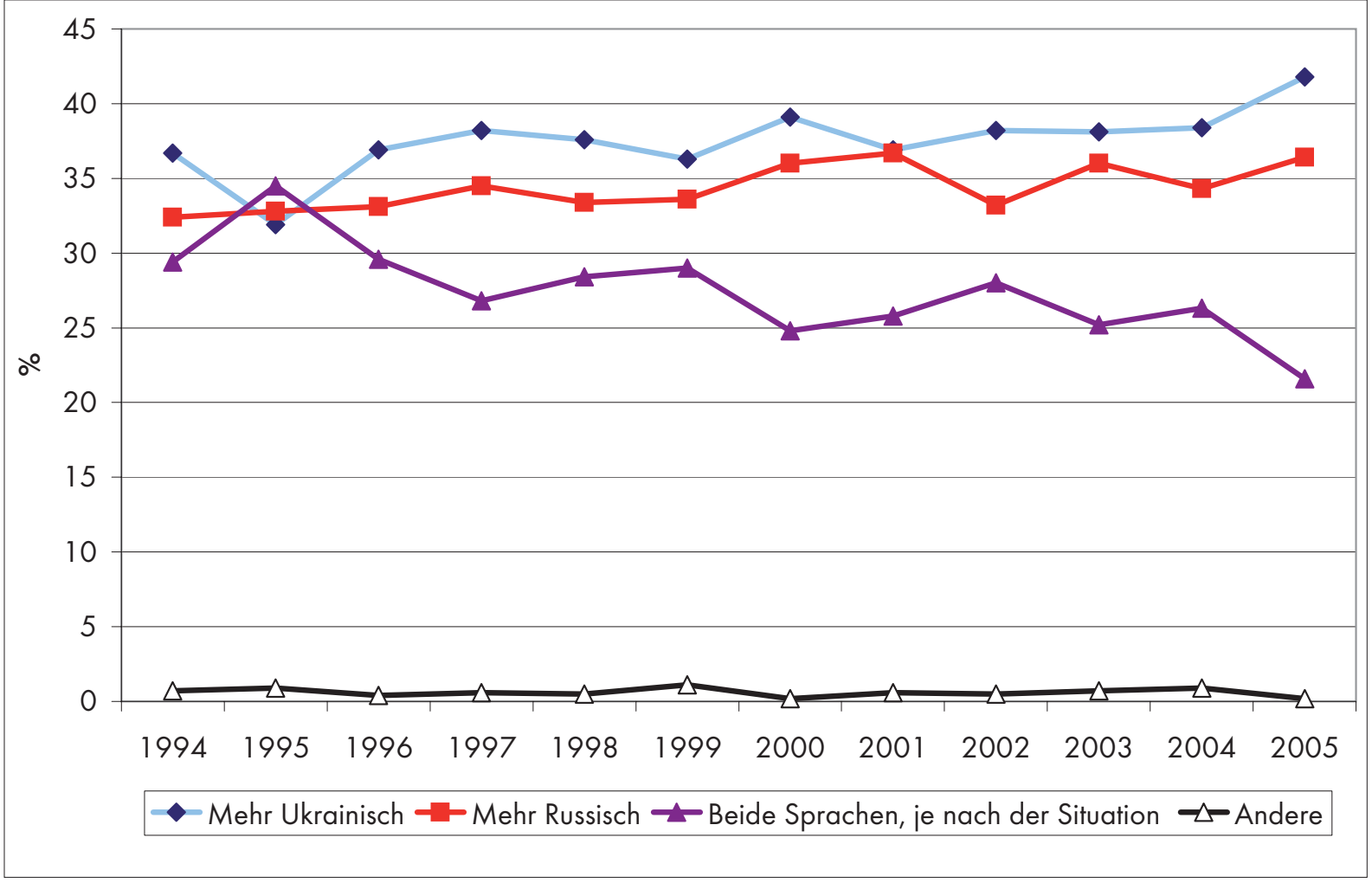

Quelle: Natalya Panina: Ukrainian Society: 1994 - 2005, Sociological Monitoring. Institute of Sociology, National Academy of Sciences of Ukraine, Kiew 2005, S. 58

Grafik 5: Unterrichtssprachen im Bildungswesen

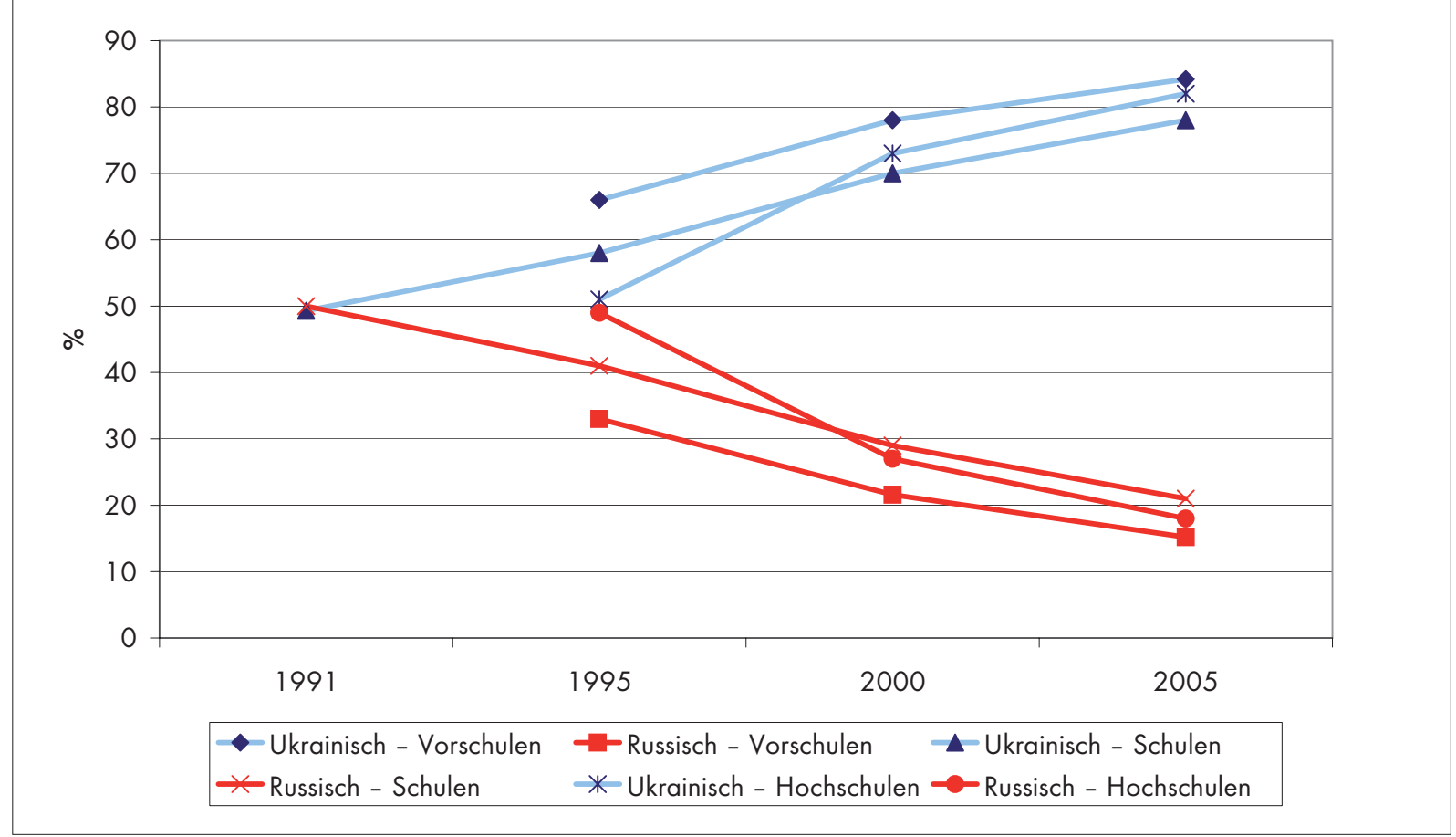

Quellen: siehe Tabellen 1 bis 3 
Tabelle 1: Ukrainisch und Russisch als Sprachen in den Vorschuleinrichtungen. Anteil der Kinder in Prozent

\begin{tabular}{|c|c|c|c|c|c|c|}
\hline \multirow{3}{*}{ Ukraine insgesamt } & \multicolumn{3}{|c|}{ Ukrainisch } & \multicolumn{3}{|c|}{ Russisch } \\
\hline & 1995 & 2000 & 2005 & 1995 & 2000 & 2005 \\
\hline & 66,0 & 78,0 & 84,2 & 33,0 & 21,6 & 15,2 \\
\hline $\begin{array}{l}\text { Autonome Republik } \\
\text { Krim }\end{array}$ & 0,2 & 2,8 & 3,6 & 99,3 & 96,3 & 95,3 \\
\hline Gebiete: Winniza & 99,9 & 100,0 & 99,9 & 0,1 & - & - \\
\hline Wolhynien & 99,0 & 100,0 & 100,0 & 1,0 & - & - \\
\hline Dnipropetrowsk & 77,0 & 84,5 & 89,2 & 23,0 & 15,5 & 10,8 \\
\hline Donezk & 10,0 & 34,5 & 65,9 & 90,0 & 65,5 & 34,1 \\
\hline Shytomyr & 98,0 & 99,8 & 100,0 & 2,0 & 0,2 & - \\
\hline Transkarpatien & 89,0 & 90,2 & 88,6 & 1,0 & 0,6 & 0,5 \\
\hline Saporishshja & 36,0 & 62,9 & 77,4 & 64,0 & 37,1 & 22,6 \\
\hline Iwano-Frankiwsk & 99,8 & 100,0 & 100,0 & 0,2 & - & - \\
\hline Kiew (Gebiet) & 97,0 & 99,6 & 99,9 & 3,0 & 0,4 & 0,1 \\
\hline Kiew (Stadt) & 99,0 & 99,9 & 99,9 & 1,0 & 0,1 & 0,1 \\
\hline Kirowohrad & 96,0 & 98,2 & 99,2 & 4,0 & 1,8 & 0,8 \\
\hline Luhansk & 14,0 & 19,7 & 35,6 & 86,0 & 80,3 & 64,4 \\
\hline Lemberg & 99,6 & 99,7 & 99,9 & 0,3 & - & - \\
\hline Mykolajiw & 71,0 & 90,4 & 96,2 & 29,0 & 9,6 & 3,8 \\
\hline Odessa & 33,0 & 62,9 & 72,9 & 66,0 & 35,4 & 25,5 \\
\hline Poltawa & 98,0 & 98,3 & 99,1 & 2,0 & 1,7 & 0,9 \\
\hline Riwne & 99,6 & 99,9 & 100,0 & 0,4 & 0,1 & - \\
\hline Sumy & 87,0 & 97,7 & 97,5 & 13,0 & 2,3 & 2,5 \\
\hline Ternopil & 100,0 & 100,0 & 100,0 & - & - & - \\
\hline Charkiw & 48,0 & 82,7 & 88,7 & 52,0 & 17,3 & 11,3 \\
\hline Cherson & 82,0 & 83,2 & 86,3 & 18,0 & 16,8 & 13,7 \\
\hline Chmelnyzkyj & 99,0 & 99,9 & 99,9 & 1,0 & 0,1 & 0,0 \\
\hline Tscherkasy & 99,6 & 99,9 & 99,9 & 0,4 & 0,1 & 0,1 \\
\hline Tscherniwzi & 92,0 & 93,6 & 91,3 & 0,5 & 0,2 & - \\
\hline Tschernihiw & 92,0 & 99,5 & 99,9 & 8,0 & 0,5 & 0,1 \\
\hline
\end{tabular}

Quelle: Statistisches Jahrbuch der Ukraine für 2005, Kiew 2006, S. 454. 
Tabelle 2: Ukrainisch und Russisch als Unterrichtssprachen an allgemein bildenden Schulen. Anteil der Schüler in Prozent

\begin{tabular}{|c|c|c|c|c|c|c|c|c|}
\hline & \multicolumn{4}{|c|}{ Ukrainisch } & \multicolumn{4}{|c|}{ Russisch } \\
\hline & 1991 & 1995 & 2000 & 2005 & 1991 & 1995 & 2000 & 2005 \\
\hline Ukraine insgesamt & 49,3 & 58 & 70 & 78 & 50,0 & 41 & 29 & 21 \\
\hline $\begin{array}{l}\text { Autonome Republik } \\
\text { Krim }\end{array}$ & & 0,1 & 0,9 & 5 & 99,96 & 99,5 & 97 & 93 \\
\hline Gebiete: Winniza & 81,3 & 91 & 98 & 99 & 18,7 & 9 & 2 & 1 \\
\hline Wolhynien & 94,6 & 98 & 99 & 99,7 & 5,4 & 2 & 1 & 0,3 \\
\hline Dnipropetrowsk & 31,1 & 46 & 68 & 78 & 68,9 & 54 & 32 & 22 \\
\hline Donezk & 3,3 & 6 & 14 & 29 & 96,7 & 94 & 86 & 71 \\
\hline Shytomyr & 76,6 & 86 & 96 & 99 & 23,3 & 14 & 4 & 1 \\
\hline Transkarpatien & 81,7 & 84 & 86 & 86 & 7,3 & 4 & 2 & 1 \\
\hline Saporishshja & 22,7 & 31 & 45 & 60 & 77,3 & 69 & 55 & 40 \\
\hline Iwano-Frankiwsk & 96,0 & 98 & 99 & 99,7 & 4,0 & 2 & 1 & 0,2 \\
\hline Kiew(Gebiet) & 84,6 & 92 & 97 & 99 & 15,4 & 8 & 3 & 1 \\
\hline Kiew(Stadt) & 30,9 & 70 & 93 & 96 & 69,0 & 30 & 7 & 4 \\
\hline Kirowohrad & 62,2 & 75 & 89 & 96 & 37,8 & 25 & 11 & 4 \\
\hline Luhansk & 6,7 & 9 & 17 & 34 & 93,3 & 91 & 83 & 66 \\
\hline Lemberg & 91,8 & 96 & 98 & 99 & 8,1 & 4 & 2 & 1 \\
\hline Mykolajiw & 43,5 & 56 & 74 & 87 & 56,5 & 44 & 26 & 13 \\
\hline Odessa & 24,5 & 32 & 47 & 65 & 73,5 & 66 & 51 & 33 \\
\hline Poltawa & 74,3 & 83 & 93 & 97 & 25,7 & 17 & 7 & 3 \\
\hline Riwne & 93,6 & 99 & 99,7 & 99,9 & 6,4 & 1 & 0,3 & 0,1 \\
\hline Sumy & 48,5 & 63 & 83 & 93 & 51,5 & 37 & 17 & 7 \\
\hline Ternopil & 97,6 & 99 & 99,7 & 99,8 & 2,4 & 1 & 0,3 & 0,2 \\
\hline Charkiw & 28,0 & 37 & 55 & 71 & 72,0 & 63 & 45 & 29 \\
\hline Cherson & 51,7 & 60 & 76 & 83 & 48,3 & 40 & 24 & 17 \\
\hline Chmelnyzkyj & 81,5 & 92 & 98 & 99,3 & 18,5 & 8 & 2 & 0,4 \\
\hline Tscherkasy & 75,8 & 86 & 96 & 98 & 24,2 & 14 & 4 & 2 \\
\hline Tscherniwzi & 67,7 & 78 & 81 & 81 & 15,7 & 5 & 2 & 0,7 \\
\hline Tschernihiw & 67,1 & 81 & 94 & 99 & 32,9 & 19 & 6 & 1 \\
\hline
\end{tabular}

Quellen: Peter Hilkes: Nationswerdung und die Ukrainisierung des Bildungswesens, in: Gerhard Simon (Hrsg.): Die neue Ukraine. Gesellschaft-Wirtschaft-Politik (1991-2001), Köln 2002, S. 160-161; Statistisches Jahrbuch der Ukraine für 2005, Kiew 2006, S. 461. 
Tabelle 3: Ukrainisch und Russisch als Unterrichtssprachen in den höheren Lehranstalten der III. und IV. Akkreditierungsstufe (Hochschulen, Akademien, Universitäten).

Anteil der Studenten in Prozent

\begin{tabular}{|c|c|c|c|c|c|c|}
\hline & & krainis & & & Russisc & \\
\hline & 1995 & 2000 & 2005 & 1995 & 2000 & 2005 \\
\hline Ukraine insgesamt & 51 & 73 & 82 & 49 & 27 & 18 \\
\hline $\begin{array}{l}\text { Autonome Republik } \\
\text { Krim }\end{array}$ & - & 0,1 & 7 & 100 & 99,9 & 93 \\
\hline Gebiete: Winniza & 85 & 99 & 99 & 15 & 1 & 1 \\
\hline Wolhynien & 97 & 100 & 100 & 3 & - & - \\
\hline Dnipropetrowsk & 32 & 62 & 79 & 68 & 38 & 21 \\
\hline Donezk & 8 & 23 & 34 & 92 & 77 & 66 \\
\hline Shytomyr & 78 & 92 & 97 & 22 & 8 & 3 \\
\hline Transkarpatien & 100 & 98 & 97 & - & - & - \\
\hline Saporishshja & 20 & 70 & 84 & 80 & 30 & 15 \\
\hline Iwano-Frankiwsk & 100 & 100 & 100 & - & - & - \\
\hline Kiew(Gebiet) & 99 & 99,8 & 100 & 0,8 & 0,2 & - \\
\hline $\operatorname{Kiew}(\operatorname{Stadt})$ & 67 & 94 & 96 & 33 & 6 & 3 \\
\hline Kirowohrad & 85 & 92 & 91 & 15 & 8 & 9 \\
\hline Luhansk & 8 & 26 & 43 & 92 & 74 & 57 \\
\hline Lemberg & 100 & 100 & 100 & - & - & - \\
\hline Mykolajiw & 42 & 91 & 99,8 & 58 & 9 & 0,2 \\
\hline Odessa & 30 & 66 & 76 & 70 & 34 & 24 \\
\hline Poltawa & 68 & 83 & 99,1 & 32 & 17 & 0,9 \\
\hline Riwne & 97 & 100 & 100 & 3 & - & - \\
\hline Sumy & 41 & 100 & 99,8 & 59 & - & 0,2 \\
\hline Ternopil & 100 & 100 & 100 & - & - & - \\
\hline Charkiw & 31 & 66 & 76 & 69 & 34 & 23 \\
\hline Cherson & 40 & 60 & 65 & 60 & 40 & 35 \\
\hline Chmelnyzkyj & 99 & 99 & 99,8 & 1 & 1 & 0,2 \\
\hline Tscherkasy & 84 & 98 & 99,8 & 16 & 2 & 0,2 \\
\hline Tscherniwzi & 99,7 & 99,9 & 100 & 0,3 & 0,1 & - \\
\hline Tschernihiw & 78 & 94 & 96 & 22 & 6 & 4 \\
\hline
\end{tabular}

Quelle: Statistisches Jahrbuch der Ukraine für 2005, Kiew 2006, S. 468. 
Grafik 6: Welche Sprachen (außer Ukrainisch) müssen Ihrer Meinung nach an Sekundarschulen unterrichtet werden? (Mehrfachantworten möglich)

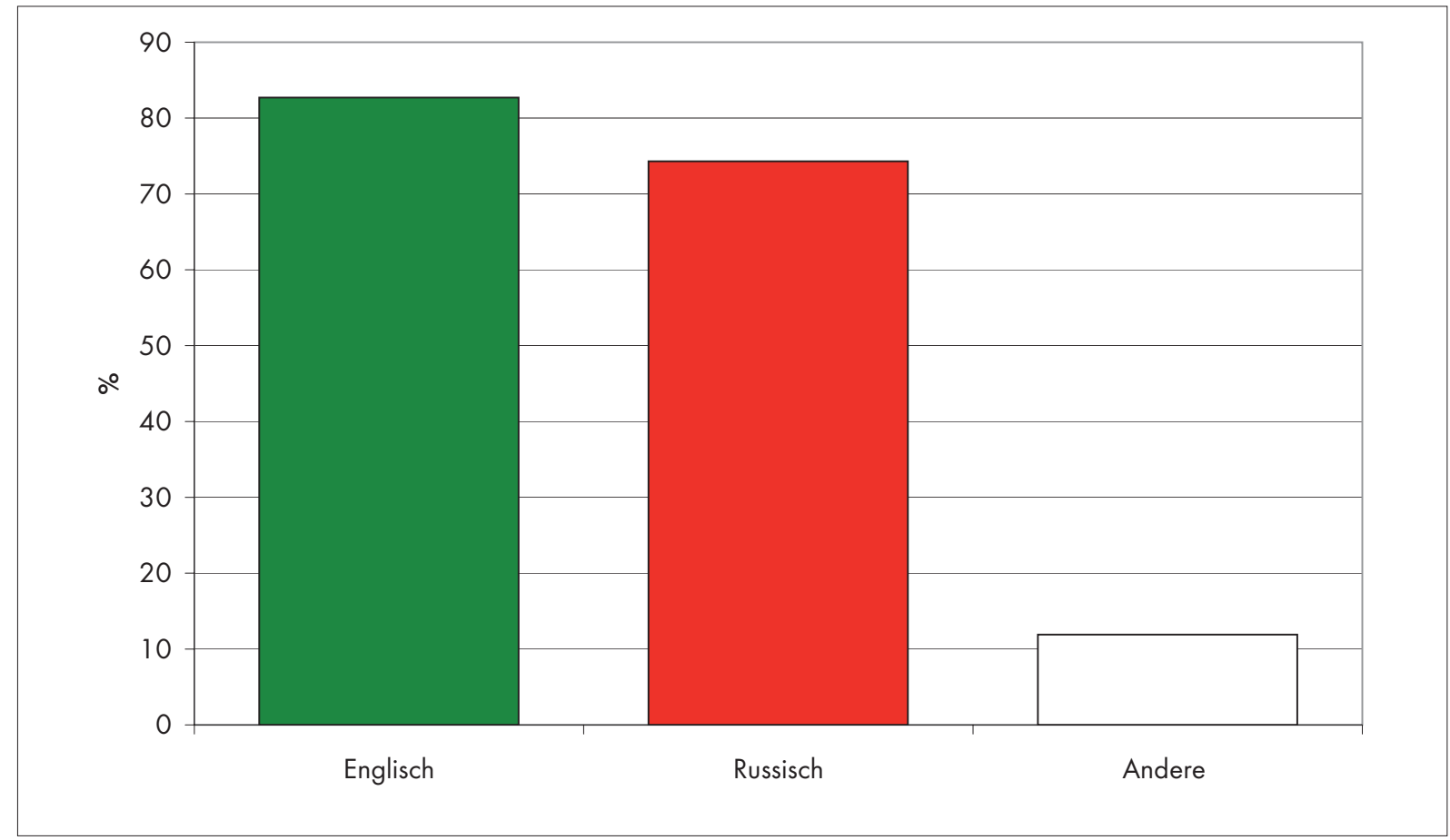

Quelle: Natalya Panina: Ukrainian Society: 1994 - 2005, Sociological Monitoring. Institute of Sociology, National Academy of Sciences of Ukraine, Kiew 2005, S. 58

Grafik 7: Glauben Sie, die russische Sprache sollte in der Ukraine einen offiziellen Status erhalten?

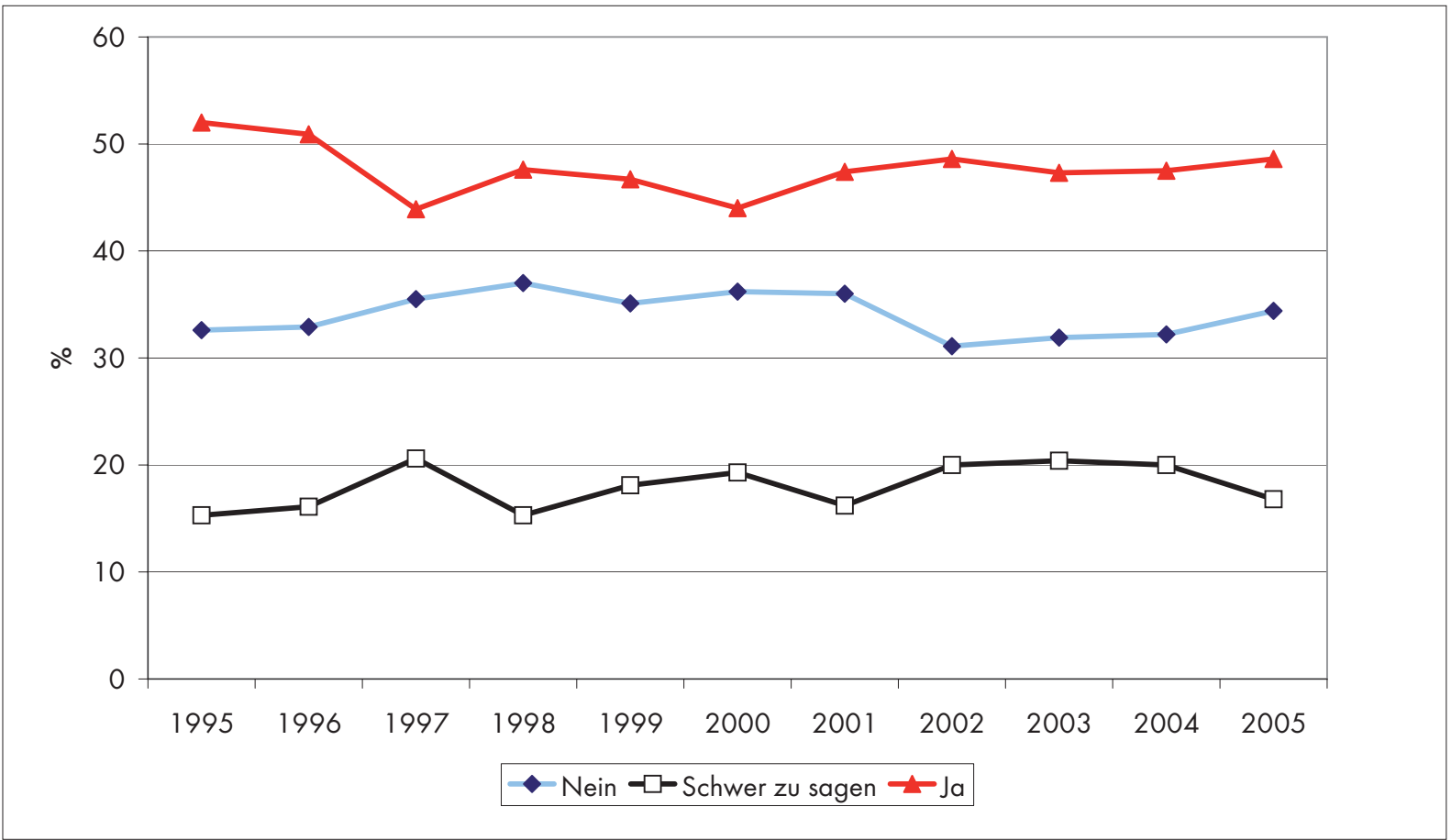

Quelle: Natalya Panina: Ukrainian Society: 1994 - 2005, Sociological Monitoring. Institute of Sociology, National Academy of Sciences of Ukraine, Kiew 2005, S. 58 


\section{Kommentar}

\section{Gespalten in Ost und West? Sprachenfrage und Geschichtspolitik in der Ukraine im Kontext der Wahlkämpfe 2004 und 2006}

Wilfried Jilge, GWZO, Leipzig

\section{Zusammenfassung:}

Trotz des gestiegenen Interesses an der Ukraine während und auch nach der „Orangen Revolution“ dominiert in den westlichen Medien immer noch das Bild eines entlang weitgehend identischer politischer und sprachkultureller Trennlinien in Ost und West gespaltenen Landes. Die Phrase von der Spaltung des Landes, das angeblich kurz vor dem Auseinanderbrechen steht, ist jedoch vor allem Teil einer politischen Herrschaftsstrategie, die in der zweiten Amtsphase von Präsident Kutschma von den Ideologen des Regimes mit subtilen und weniger subtilen Mitteln (Zensur) zur Legitimation einer autoritären Machtvertikale erdacht wurde.

\section{Ost-West-Stereotypenketten als Wahl- kampfstrategie der Staatsführung bei den Präsidentenwahlen 2004}

\section{$\mathrm{D}$} ie symbolische Politik und Propaganda der Staatsführung unter Präsident Kutschma und Premierminister und Präsidentschaftskandidat Janukowitsch hat im Wahlkampf des Jahres 2004 - massiv unterstützt von gelenkten Medien und „administrativen Ressourcen" - das Bild von der Ost-West-Spaltung der Ukraine geschickt genutzt und verstärkt. Sie konstruierte entlang vermeintlich sprach- und erinnerungskultureller Gegensätze eine emotional aufgeladene, das Land polarisierende Ost-West-Stereotypenkette: Auf der einen Seite wurde der Herausforderer Viktor Juschtschenko sowie die ihn unterstützenden Kräfte zu Repräsentanten eines radikal antirussischen, ukrainischsprachigen „westlichen“ und "galizischen“ $\mathrm{Na}$ tionalismus stilisiert, die Politik im Dienste Amerikas betreiben und das Land sozial und politisch spalten wollten. Der Kandidat der Staatsführung präsentierte sich dagegen als pragmatische, mit Russland „konstruktive Beziehungen" pflegende politische Kraft, die sich für die kulturellen Rechte der russischsprachigen Bevölkerung in einem „bilingualen“ Land einsetzte und damit die große Mehrheit des „gesunden Menschenverstandes“ gegenüber den angeblichen „westukrainischen Radikalen" präsentierte. Zentrale Grundlage dieser Kampagne war die symbolische Besetzung des Mythos vom „Großen Vaterländischen Krieg“ und eine aus diesem Mythos abgeleitete resowjetisierende Symbolpolitik, die streckenweise wie eine staatliche Hetzkampagne sowjetischen Typs anmutete.

Besonders in den überwiegend russischsprachigen Gebieten der Ostukraine oder auf der Halbinsel Krim wurden die Repräsentanten der Opposition, analog zur stalinistischen Freund-Feind-Propaganda der 1940er Jahre, als radikale "Nationalisten“ und „Faschisten“ gebrandmarkt. Diese "Nationalisten" seien gleich- sam als „Okkupanten“ eingedrungen, um als vom amerikanischen Imperialismus gekaufte Kapitalisten die ökonomischen Grundlagen der Bevölkerung zu zerstören und die russische Sprachkultur in der Ukraine auszurotten. Die symbolische Politik gegen die "Naschisten" (eine pejorative Bezeichnung für die Anhänger von Viktor Juschtschenkos Bewegung "Nascha Ukraina“ mit deutlichem Anklang an „Nazis“ und „Faschisten“) zog sich im Herbst 2004 wie ein roter Faden durch die Wahlkampfkampagne des Regimes, in der sich die geschichtspolitische Agitation gegen die "Okkupanten" mit antiwestlichen Stereotypen und Antiamerikanismus verband. Kurz vor der Stichwahl am 21. November (2. Wahlgang) wurden an die Haushalte in Donezk und anderen Regionen massenhaft anonyme Broschüren verteilt, in denen Juschtschenko als amerikanischer Onkel Sam abgebildet war, der sich an den Wähler mit der Frage „Bist Du zum Bürgerkrieg bereit?" wendete. Juschtschenko sollte als galizischer, ,uniatischer Nationalist gebrandmarkt werden, der die Einheit des Staates gefährdet und das Land ins Chaos stürzt. Traurige Berühmtheit hat auch ein Wahlspot im Fernsehen erlangt, in dem ein Cowboy Juschtschenko auf ihr herumreitend die Karte der Ukraine in Brand setzt, wozu eine sowjetpatriotische Melodie mit dem Refrain ,alles, nur kein Krieg" erklang.

Es gibt allerdings einigen Grund anzunehmen, dass gerade der Versuch der Staatsführung, das Land zu spalten, viele Menschen erst dazu getrieben hat, sich der demokratischen Protestbewegung anzuschließen. „Schid i Zachid rasom“, Ost und West gemeinsam, war eine der wichtigsten Losungen des Kiewer Majdan. Dieses ukrainisch-patriotische, aber inklusive Selbstverständnis der überwiegenden Mehrheit der Anhänger der friedlichen Proteste wurde geradezu programmatisch in einem Song der PunkrockGruppe „Tartak“ (deutsch: Sägewerk) mit dem Titel 
„Ich will nicht“ ausgedrückt: In diesem Song erklärt „Tartak“, dass die „nationale Idee“ zu einem „Mittel der Spekulation“ verkommen sei. Gleichzeitig entlarvt der Liedtext die Divide-et-Impera-Politik des herrschenden Regimes: „Tatsächlich ist es einfach, das Leben zu verändern! ... Einfach auf die Straße gehen, den Müll aufräumen, sein Land lieben, ... und sich als Teil eines einigen Volkes fühlen ... eine Gemeinschaft unabhängiger Menschen ... ohne ,Linksufrige` und ohne, Rechtsufrige““.

\section{Das Spektrum nationaler und sprachkultu- reller Identitäten in der Ukraine}

Tnterschiedliche Gebiete der heutigen Ukraine gehörten in verschiedenen historischen Perioden zu unterschiedlichen Großreichen, wie dem Russischen Reich, Österreich-Ungarn, Polen-Litauen oder dem Krim-Chanat. So haben sich die heutigen ukrainischen Regionen in unterschiedlicher Weise historisch entwickelt. In der Westukraine, die im 19. Jh. und Anfang des 20. Jh. Teil Österreichs-Ungarns war, entstand eine starke ukrainisch-nationale Bewegung. Zudem geriet die Westukraine erst später unter die sowjetische Herrschaft als die übrigen Landesteile. Die Basis der ukrainischen Nationalbewegung lag daher vor allem im Westen, wo die ukrainische Sprache und ein ethnisch-ukrainisches Nationalbewusstsein besonders stark verbreitet waren.

Die Unterschiede in den historisch-politischen Traditionen werden zudem durch sprachliche Trennlinien verstärkt. In den östlichen Regionen, die zum Russischen Reich gehörten und früher als die westlichen Regionen in die Sowjetunion inkorporiert wurden, ist die Verwendung des Russischen und russisch-kulturelle Orientierungen weitaus stärker verbreitet. Es wäre jedoch ein Fehler anzunehmen, die Ukraine ginge in einer statischen Ost-West-Dichotomie auf, die das Land in einen russischsprachigen, sowjetnostalgischen und eher an autoritären Werten orientierten Osten einerseits und in eine - auch noch wesentlich kleinere - westliche, nationaldemokratische Westukraine andererseits teilt. Die Sprachenfrage führt vor allem auf der Ebene der Debatten der ukrainischsprachigen und russischsprachigen Eliten zu Spannungen. Ernsthafte gesellschaftliche Spannungen sind bisher ausgeblieben. Freilich haben die Wahlkämpfe 2004 und 2006 gezeigt, dass die Sprachenfrage zur politischen Mobilisierung und Emotionalisierung gesellschaftlicher und politischer Konflikte instrumentalisiert werden kann und deshalb nicht unterschätzt werden darf.

Laut der ukrainischen Volkszählung von 2001 leben in der Ukraine etwa 37,5 Millionen ethnische Ukrainer, die 77,8\% (1989: ca. 73\%) der etwa 48 Millionen starken Gesamtbevölkerung ausmachen. Die größte Minderheit sind Russen 17,3\% (1989:
$22,1 \%)$ gefolgt von Weißrussen $(0,6 \%)$, Moldauern, Krimtataren (0,5\%), Bulgaren (0,4\%), Ungarn, Rumänen, Polen $(0,3 \%)$ und Juden $(0,2 \%)$ sowie anderen Minderheiten. Von der Gesamtbevölkerung gaben dabei $67,5 \%$ Ukrainisch als Muttersprache an, also etwa 2,8\% mehr als 1989 . Von den Ukrainern nannten $85,2 \%$ Ukrainisch als Muttersprache. Allerdings können solche Daten irreführend sein, da die genannte Muttersprache nicht die im Alltag vorrangig verwendete Sprache sein muss. Zu Recht ist außerdem darauf hingewiesen worden, dass viele Ukrainer und einige Russen bilingual sind und die Grenzen zwischen den drei größten linguistischen Gruppen (ukrainischsprachige Ukrainer, russischsprachige Ukrainer, russischsprachige Russen) damit häufig fließend sind. Nach Khmelko gab es unter der erwachsenen Bevölkerung im Jahr 2003 47,6\% ukrainischsprachige Ukrainer, $30,4 \%$ russischsprachige Ukrainer und $14,8 \%$ russischsprachige Russen. In den südöstlichen Regionen, in denen mehrheitlich Russisch gesprochen wird, wird die Vermittlung von ukrainischer Sprache und Kultur jedoch keineswegs als Oktroy empfunden. Vielmehr wird die Selbstdefinition als Ukrainer mit einer positiven Haltung zur russischen Sprachkultur verbunden und eine eigene ukrainische, aber vom ukrainischen Westen unterschiedene, vor allem am Kosakentum orientierte Tradition entwickelt. Sprachliche und nationale Identität sind in der Ukraine also häufig nicht deckungsgleich.

Selbst in dem russischsprachigen und schwerindustriell geprägten Gebiet Donezk, wo die Mehrheit der Bevölkerung vor allem an engen Beziehungen zu Russland interessiert ist, sind die Verhältnisse komplexer als sie auf den ersten Blick scheinen: Bereits in den 1990er Jahren kamen ukrainisch/US-amerikanische sozialwissenschaftliche Untersuchungen zur Stadt Donezk zu dem Ergebnis, dass zwischen 1994 und 1999 eine sowjetisch eingefärbte, aber vornehmlich auf die Region konzentrierte Identität die wichtigste Bedeutung erlangte und eine ethnischrussische Identität stärker zurücktrat. Das in der regionalen Identität dominierende, ausgesprochen selbstbezogene Selbstbild von der mächtigen, führenden Industrieregion steht in einer hohen Kontinuität zur Sowjetzeit, was von den mit der Partei der Regionen (PR) verbundenen herrschenden Klans zur Legitimation der Bewahrung überkommener wirtschaftlicher Strukturen und damit der eigenen politischen Macht genutzt wird; jedoch steht diese Identität nicht im direkten Gegensatz zur ukrainischen Identität: Die Identifikation mit der russischen und ukrainischen Identität wird gleichermaßen akzeptiert. Anders als im Westen der Ukraine spielen sozioökonomische Faktoren eine deutliche größere Rolle als sprachkulturelle Faktoren. 
Die Mehrheit der Donezker scheint die eigene Zukunft in Kiew, nicht in Moskau zu sehen. Tatsache ist jedenfalls, dass die von Janukowitschs Politstrategen im Dezember 2004, also nach dem Gerichtsbeschluss zur Wiederholung der gefälschten Präsidentschaftswahl, initiierten separatistischen Bewegungen (Beschluss zur Gründung einer „Südöstlichen Ukrainischen Autonomen Republik" auf einem Kongress in Sewerodonezk) schnell in sich zusammenbrachen und diese extreme Richtung nicht weiterverfolgt wurde. Ein Grund für das darin zum Ausdruck kommenden Bekenntnis zu „einer" Ukraine liegt auch darin, dass die östlichen und westlichen Teile der Ukraine nicht erst seit Erlangung der staatlichen Unabhängigkeit 1991, sondern in administrativer Hinsicht bereits in der Zeit der ukrainischen Sowjetrepublik, dann aber vor allem auch während der Kutschma-Ära weiter zusammengewachsen waren.

\section{Politische Wahlentscheidungen jenseits der sprachkulturellen Trennlinien}

C ine Analyse der Ergebnisse der Wiederholung des zweiten Wahlgangs der Präsidentschaftswahlen am 26.12.2004 zeigt zwar, dass „russisch-“ bzw. „ukrainischsprachig" in Verbindung mit geopolitischen Orientierungen (z.B. Haltung zur NATO) zweifellos ein wichtiges Kriterium zu Unterscheidung zwischen dem Elektorat Juschtschenkos einerseits und der Wählerschaft Janukowitschs andererseits darstellt. Eine andere Frage ist, ob daraus folgt, dass Sprache ein vorrangiges Kriterium für die Wahlentscheidung war. Von Juschtschenkos Wählern waren 92,2\% ethnische Ukrainer (Russen: 5,3\%), während sich unter den Anhängern Janukowitschs nur 60,2\% ethnische Ukrainer befanden (Russen: 33\%). Innerhalb der Wählerschaft Juschtschenkos war der Anteil ukrainischsprachiger Wähler um ein Dreifaches höher als der Anteil russischsprachiger Wähler. Dies stellte sich innerhalb der Wählerschaft Janukowitschs genau umgekehrt dar, allerdings mit weit extremeren Unterschieden: Der Anteil ukrainischsprachiger Wähler war hier mit 8,8\% minimal und lag um mehr als das Zehnfache niedriger als der Anteil russischsprachiger Wähler (91,2\%). Dem ukrainischsprachigen und nationaldemokratischen Kandidaten Juschtschenko gelang es demnach deutlich besser, in russischsprachige Wählerschichten vorzustoßen als dies umgekehrt bei Janukowitsch hinsichtlich der ukrainischsprachigen Wähler der Fall war, wodurch deutlich wird, dass vor allem ein Kandidat - nämlich Janukowitsch - polarisierte, und der andere - Juschtschenko - Potenzial zur Einigung des Landes hatte. Das knappe Viertel russischsprachiger Bürger unter Juschtschenkos Wählern war zentrale Voraussetzung für seinen Wahlsieg. Mit Viktor Juschtschenko gelang es erstmals in der Geschichte der unabhängigen
Ukraine einem ukrainischsprachigen Kandidaten mit demokratischen Reformprogramm und einem klaren außenpolitischen Bekenntnis zur euro-atlantischen Integration, Mehrheiten in der überwiegend russischsprachigen östlichen Zentralukraine zu erringen. In den nordöstlichen Gebieten Sumy und Tschernigow sowie in Poltawa erhielt Juschtschenko eindeutige Mehrheiten (79,45\%; 71,15\%; 66,00\%). Die Gebiete der östliche Zentralukraine verfügten zudem über eine ausgesprochen aktive Protest- und Oppositionsbewegung: Die ersten Zelte der Studenten wurden nicht auf dem Unabhängigkeitsplatz in Kiew, sondern bereits vor der ersten Stichwahl am 22.11.2004 in Sumy aufgeschlagen. Hintergrund waren die massiven Wahlfälschungen in der östlichen Zentralukraine. Sie können auch als Indikator dafür gewertet werden, wie umkämpft die östliche Zentralukraine zwischen dem „orangen“ und „blauen“ Lager war.

Die Bedeutung der sich allmählich abzeichnenden politischen Veränderungen in den Regionen wird vor allem im Vergleich mit den Ergebnissen früherer Wahlen deutlich: In der Stichwahl der Präsidentenwahlen vom Juli 1994 errang der Kandidat Leonid Kutschma auch in der östlichen Zentralukraine gegen den von den nationaldemokratischen Kräften unterstützten amtierenden Präsidenten Leonid Krawtschuk einen deutlichen Sieg, wobei er mit einem hinsichtlich der Sprachenfrage und der Außenpolitik dezidiert russophilem Programm antrat (Gebiet Poltawa: 59,2\% für Kutschma/37,4\% für Krawtschuk; Gebiet Sumy: 67,8\%/28,9\% und Gebiet: Tschernigow: 72,3\%/25,1). Aber auch im Jahre 2004 verhielten sich die Wähler selbst im Süden der Ukraine (Gebiete Odessa, Nikolajew, Cherson, Zaporishshja, Autonome Republik Krim), der insgesamt mit einer überwiegenden Mehrheit für den prorussischen Kandidaten Janukowitsch stimmte und wo die überwiegende Mehrheit der Bevölkerung eher die russische gegenüber der ukrainischen Sprache bevorzugt $(84,5 \%$ der Bevölkerung sind eher russischsprachig), nicht nach einem einfachen Ost-West-Schema: Im südlichen Gebiet Cherson siegte Janukowitsch zwar mit 51,32\%, aber Juschtschenko erhielt immerhin 43,43\% der Stimmen. Dass die Zustimmung zur „orangen“ Staatsführung bzw. zu den „orangen“ Parteien im Verlauf der Jahre 2005-2006 sank, nicht zuletzt aufgrund des politischen Versagens dieser politischen Kräfte, steht auf einem anderen Blatt.

Insgesamt zeigen die Wahlergebnisse der Präsidentschaftswahlen 2004, dass die Mehrheitsfähigkeit eines demokratischen, mit gemäßigten ökonomischen Reformen verbundenen und an westlichen Grundwerten orientierten Wahlangebots, repräsentiert durch einen ukrainischsprachigen Kandidaten des patriotischen Lagers, in den letzten 
Jahren von Westen nach Osten vorgerückt ist und seine Attraktivität in keiner Weise durch sprachlich-kulturelle Faktoren zwingend eingeschränkt ist. Die Faktoren Sprache und Geschichtspolitik dienen vor allem der Emotionalisierung und Mobilisierung, sind aber für politische Wahlentscheidung keineswegs ausschlaggebend: Der sprunghafte Anstieg der Zustimmung zu Premierminister Janukowitsch in den Umfragen im Oktober 2006 resultierte vor allem aus einer haushaltspolitisch fragwürdigen, von den zensierten Medien aber massiv popularisierten Erhöhung der Pensionen.

Die Tatsache, dass sich demokratische Orientierungen vom Westen des Landes in weiter östliche und südlich gelegene (eher russischsprachige) Gebiete der Ukraine ausbreiten, wurde im Grunde von den Parlamentswahlen im Jahre 2006 bestätigt. Zwar wurde die Partei der Regionen unter Viktor Janukowitsch stärkste Partei (32,14\%), eine Regierung konnte sie schließlich aber nur mit den von ihr ungeliebten Kommunisten $(3,66 \%)$ und nach dem unerwarteten Schwenk der Sozialisten (5,69\%) bilden. Am 26. März 2006 erhielten diejenigen politischen Kräfte die Mehrheit, die 2004 die „orangene Revolution“ und damit Viktor Juschtschenko unterstützt hatten: Nascha Ukraina (13,95\%), Block Julia Timoschenko (22,29\%), Sozialistische Partei der Ukraine (SPU). Die SPU, die ebenfalls zu den Parteien des „Majdan“ gehört hatte, war zuvor in der präsidentenfreundlichen Regierung Jechanurow vertreten und hat sich im Wahlkampf ausdrücklich als Regierungspartei präsentiert. Trotz massiver innenpolitischer Enttäuschungen über die ausgebliebene nachhaltige Bekämpfung der Korruption, den Umstand, dass die Verantwortlichen für die Wahlfälschungen nicht zur Verantwortung gezogen wurden, und besonders über die unterlassenen ökonomischen Reformen und ausgebliebenen sozioökonomischen Verbesserungen haben die Wähler denjenigen Parteien ihr Vertrauen ausgesprochen, die für demokratische Reformen und eine nationale ukrainische Politik standen.

\section{Gespaltene Erinnerungskulturen?}

ie Strategie des Regimes unter Kutschma scheiterte nicht nur in sprachkultureller, sondern auch geschichtspolitischer Hinsicht. Das Thema des Zweiten Weltkrieges als zentrale geschichtspolitische Konstruktionsressource einer Ost-West-Stereotypen-Kette hat offenbar auch deshalb nicht ausreichend beim Wähler verfangen, weil sich ein erheblicher Teil der Bevölkerung nicht mit den offiziellen Geschichtsbildern identifizierte. Der zentrale Konflikt in der öffentlichen Kontroverse um den Zweiten Weltkrieg ist die Debatte um die gesetzliche Rehabilitierung des antisowjetischen Untergrunds, vor allem der der Ukrai- nischen Aufstandsarmee (UPA) und die Zuerkennung des Veteranenstatus mit allen dafür vorgesehenen sozialen Vergünstigungen, d.h. ihre Gleichstellung mit den gesellschaftlich insgesamt hoch angesehenen sowjetischen Kriegsveteranen.

Die These von einer "gespaltenen Erinnerungskultur" mag im Hinblick auf die unversöhnlichen Standpunkte zwischen nationalistischen und einigen nationaldemokratischen Politikern und UPAVeteranen einerseits und linken Politikern und sowjetischen Veteranen andererseits ihre Berechtigung haben. Differenziert nach Makroregionen ist das Bild für die gesamte Ukraine aber erheblich komplizierter: Laut Umfragen des Instituts für Politik (Kiew) sprachen sich 2002 für eine Anerkennung der UPA nur 33\% der Befragten aus, während 38\% dagegen waren; aber immerhin 29\% waren unentschlossen. Die negative Bewertung der UPA erlaubt jedoch keine voreiligen Schlüsse zur gesamten nationalen Erinnerungskultur und Identität, auch wenn nationale Intellektuelle und Historiker die Anerkennung der OUN und UPA als „Helden“ zum Ausweis nationaler Gesinnung stilisieren. In der Südukraine (Gebiete Odessa, Cherson, Nikolajew) beispielsweise wird die Anerkennung der UPA von einer deutlichen Mehrheit abgelehnt. Die Figur des ukrainischen Nationalhistorikers Michailo Hruschewski wird aber von einer ebenso deutlichen Mehrheit positiv eingeschätzt. In der Zentralukraine sind die Befürworter der UPA-Anerkennung ebenfalls in einer Minderheit, während andererseits etwa $79 \%$ den Tag des Sieges für einen herausragenden Feiertag halten (Westen: 28\%, Osten: 84\%, Süden: $70 \%)$. Das in der Zentralukraine häufig noch vom sowjetischen Feiertagskalender geprägte Gedenken an die für den Sieg im „Großen Vaterländischen Krieg“ gefallenen „Söhne der Ukraine“ verbindet sich mit christlichen Motiven und Riten sowie nationalen Konnotationen, indem auf die Bedeutung des Sieges über den Faschismus für den Bestand der Ukraine und ihre internationale Anerkennung hingewiesen wird. Die Erinnerungskulturen der Ukraine gehen daher nicht in einem Gegensatz zwischen einer prosowjetischen und antisowjetischen nationalen Erinnerungskultur auf. Das Gedenken an den „Sieg im Großen Vaterländischen Krieg“, bei dem häufig noch auf sowjetische Rituale und Symbole zurückgegriffen wird, ist kein hinreichender Indikator für mangelnde nationale Identifikation oder gar das Fehlen demokratischer Werte.

Das vielschichtige Bild der Kriegserinnerung resultiert u.a. aus tatsächlich widersprüchlichen historischen Kausalitäten und widersprüchlichen Erfahrungen einzelner Personen und ethnischer und sozialer Gruppen, wobei sich diese unterschiedlichen Erfahrungen oft innerhalb ein und derselben Familie finden. In 
einem nationalen Geschichtsbild, das beispielsweise die Rolle und den von Teilen der Bevölkerung empfundenen Befreiungscharakter der Roten Armee durch selektive Fixierung auf ihre Verbrechen und deren Gleichsetzung mit den NS-Verbrechen zugunsten eines „sauberen“ Heldengeschichtsbildes der nationalistischen Organisationen entmoralisieren und völlig entwerten will, haben diese Widersprüche ebenso wenig Platz wie in der sowjetisch eingefärbten, gegen die „nationalistische Opposition“ gerichtete Hetzkampagne gegen Präsidentschaftskandidat Juschtschenko 2004.

\section{Die Statusfrage des Russischen als macht- politisches Instrument (2005/2006)}

$\mathrm{D}$ ie Partei der Regionen und Vorsitzender Viktor Janukowitsch haben aus der Wahlniederlage bei den Präsidentschaftswahlen 2004 durchaus Konsequenzen gezogen: Im Parlamentswahlkampf Anfang 2006 präsentierte sich ihr Spitzenkandidat als Garant der staatlichen Einheit auf der Basis einer dezent in ukrainische Nationalfarben (blau-gelb) und Parteifarben (blau-weiß) eingekleideten visuellen Symbolpolitik. Gleichwohl hat vor allem die Partei der Regionen nach den verlorenen Präsidentenwahlen 2004 und während des Parlamentswahlkampfes 2006 die machtpolitische Instrumentalisierung der Sprachenfrage immer wieder betrieben, indem sie mit regionalen Initiativen zur Anerkennung des Russischen als „offizieller Sprache“ die Bevölkerung vor allem in den östlichen und südlichen Regionen sowie auf der Halbinsel Krim gegen die nationalistischen „orangenen Chaoten“ zu mobilisieren suchte.

Die derzeitige Parlamentsmehrheit der Regierung Janukowitsch ließ Anfang des Jahres 2007 ein das Gesetzesprojekt „Über die Sprachen der Ukraine“ registrieren, das den Begriff der „regionalen Sprache“ einführt und diese Sprache im Gebrauch bei Behörden mit der ukrainischen Staatssprache gleichstellt. Das Gesetz erhält auf den ersten Blick demokratische Ansätze und konstruktive Fortschritte gegenüber der gegenwärtigen Lage: Eine Sprache in einem bestimmten Territorium kann zur regionalen Sprache erklärt werden, wenn mindestens $10 \%$ der Bevölkerung zu dieser Sprachgruppe gehören. Tatsächlich aber zielt das Gesetz auf eine einseitige Begünstigung des Russischen gegenüber dem Ukrainischen und anderen Sprachen in den von der PR dominierten südlichen und östlichen Regionen, da die Festlegung einer Regionalsprache von der Mehrheit des Gebietsparlaments beschlossen werden muss und Verfahrensfragen (sprachliche Qualifikation der Beamten) nicht völlig geklärt sind. Es ist derzeit beispielsweise schwer vorstellbar, dass die von der PR geführte Mehrheit im Parlament der Autonomen Republik Krim dem Krimtatarischen den Status einer Regionalsprache zuerkennt. Insofern dient die Sprachenfrage in diesem Fall eher Festigung der Position der PR in den östlichen und südlichen Landesteilen als einer langfristigen, von allen politischen Kräften akzeptierten und pragmatischen Lösung. Ähnliches gilt auch für Teile der nationaldemokratischen Opposition, die in jeder Änderung des Status quo eine Bedrohung der Existenz der ukrainischen Nation sehen.

Die Frage der Verwendung der stark verwandten ukrainischen und russischen Sprachen dürfte nicht das Hauptproblem der Distanz der Regionen untereinander sein. Größere Bedeutung dürften strukturelle Faktoren haben, die die Kommunikation der Regionen untereinander erschweren: Eine regional versäulte Printmedienlandschaft, die den Zugang zu allukrainischen Medien in den Provinzen häufig unmöglich macht, ein völlig ineffizientes Distributionssystem auf dem Buchmarkt oder eine Verkehrsinfrastruktur, die die öffentliche Verbindung selbst zwischen den Gebietszentren innerhalb einer Großregion erheblich erschwert. Sowohl die Partei der Regionen als auch die nationaldemokratische Opposition haben bisher keine nachhaltige Debatte zu diesen Strukturproblemen angestoßen und kaum Konzepte vorgelegt, die den in der Gesellschaft der Ukraine seit einiger Zeit verstärkt zu beobachtenden Prozess einer politischen und demokratischen Nationsbildung fördern könnten. Insofern gilt hier Ähnliches wie im Bereich des allgemeinen Demokratisierungsprozesses: Die Gesellschaft scheint schon weiter zu sein als ihre politischen Eliten.

Über den Autor

Wilfried Jilge M.A ist, wissenschaftlicher Mitarbeiter am Geisteswissenschaftlichen Zentrum Geschichte und Kultur Ostmitteleuropas (GWZO) Leipzig.

Lesetipps

- Wilfried Jilge: Staatssymbolik und Nationsbildung in der Ukraine (1991-2001). In: Ost-West. Europäische Perspektiven 2 (2001), H. 1.

- Wilfried Jilge: The Politics of History and the Second World War in Post-Communist Ukraine (1986/1991-2004/2005). In: Wilfried Jilge/ Stefan Troebst (Hrsg.): Gespaltene Geschichtskulturen? Kollektive Erinnerungskulturen und Zweiter Weltkrieg in der Ukraine. Stuttgart 2006 (=Themenheft Jahrbücher für Geschichte Osteuropas 54 (2006)), H. 1, S. 50-81.

- Gerhard Simon: Neubeginn in der Ukraine. Vom Schwanken zur Revolution in Orange. In: Osteuropa 55 (2005), H. 1, S. 16-33.

- Susan Stewart: Sprachenpolitik als Sicherheitsproblem in der Ukraine. MZES-Arbeitspapiere, Nr. 20. Mannheim 2000.

- Kerstin Zimmer: Machteliten im ukrainischen Donbass. Bedingungen und Konsequenzen der Transformation einer alten Industrieregion. Berlin 2006 (= Gesellschaftliche Transformationen, 12), S. 159-164. 


\section{Chronik}

\section{Vom 22. Januar bis zum 13. Februar 2007}

22.1.2007 $\quad$ Die EU-Außenminister einigen sich bei einem Treffen in Brüssel auf die Aufnahme von Verhandlungen mit der Ukraine über ein neues Partnerschaftsabkommen sowie ein mögliches Freihandelsabkommen. Eine indirekte Bezugnahme auf die Beitrittsabsichten der Ukraine, die u.a. Polen befürwortete hatte, wird nicht in die abschliessende Erklärung aufgenommen.

22.1.2007 In Simferopol auf der Halbinsel Krim protestieren etwa 4.000 Krimtataren gegen eine Verschärfung der Strafen für illegale Landbesetzung. Die Krimtataren wollen mit Landbesetzungen ihre historischen Eigentumsansprüche geltend machen.

23.1.2007 $\quad$ Präsident Viktor Juschtschenko entlässt den ukrainischen Botschafter in Österreich, Wolodimir Jeltschenko, da dieser zwei Oppositionsführern aus Turkmenistan auf Einladung des ukrainischen Transportministers Mikola Rudkowski Visa erteilt habe. Dies sei eine Einmischung in die ukrainische Außenpolitik. Juschtschenko forderte Ministerpräsident Viktor Janukowitsch auf, über die Entlassung Rudkowskis nachzudenken.

26.1.2007 Das Privatisierungsprogramm für 2007 wird von Präsident Viktor Juschtschenko genehmigt. Die Privatisierungseinnahmen sollen sich auf über 2 Mrd. US-Dollar belaufen.

30.1.2007 Außenminister Boris Tarasjuk erklärt seinen Rücktritt. Amtierender Aussenminister wird sein bisheriger erster Stellvertreter Wolodimir Ohrizko. Das Parlament hatte bereits im Vorjahr für seine Entlassung gestimmt, die Präsident Viktor Juschtschenko jedoch nicht akzeptiert hatte. Tarasjuk war so zum Kristallisationspunkt des Machtkampfes zwischen Präsident und Regierungskoalition geworden.

2.2.2007 Parlamentspräsident Oleksandr Moroz veröffentlicht das Gesetz über das Ministerkabinett in den Amtsblättern. Trotz der Weigerung des Präsidenten tritt das Gesetz damit formal in Kraft. Präsident Viktor Juschtschenko erklärt, dass er das Verfassungsgericht anrufen werde, da sowohl Teile des Gesetzes als auch das Verfahren seiner Inkraftsetzung verfassungswidrig seien.

5.2.2007 $\quad$ Präsident Viktor Juschtschenko schlägt dem Parlament den bisherigen ersten stellvertretenden Aussenminister Wolodimir Ohrizko als Nachfolger von Boris Tarasjuk vor. Ohrizko hatte erklärt, den Kurs von Tarasjuk unverändert fortsetzen zu wollen.

6.2.2007 Das Parlament stimmt mit großer Mehrheit für ein Gesetz, das die staatliche Kontrolle über das nationale Erdgaspipelinenetz festschreibt.

6.2.2007 Als Leiter des Geheimdienstes schlägt Präsident Viktor Juschtschenko dem Parlament Viktor Korol vor, der der Fraktion von Unsere Ukraine angehört.

8.2.2007 $\quad$ Präsident Viktor Juschtschenko besucht Deutschland, wo er sich u.a. mit Bundeskanzlerin Angela Merkel trifft.

9.2.2007 Präsident Viktor Juschtschenko kritisiert die von der Werchowna Rada in erster Lesung angenommenen Veränderungen im Gesetz „Über das Ministerkabinett“.

10.02.2007 Das Ministerkabinett der Ukraine legt die Quote für die Immigration in die Ukraine im Jahr 2007 auf 7.223 Menschen fest.

10.02.2007 Auf der Sicherheitskonferenz in München erklärt Präsident Viktor Juschtschenko, dass die Ukraine mehr Erdöl und -gas aus Russland durch ihre Transitpipelines befördern könne.

12.02.2007 Präsident Viktor Juschtschenko bestätigt die nationale Sicherheitsstrategie der Ukraine.

13.02.2007 Präsident Viktor Juschtschenko erklärt auf dem jährlichen Treffen mit den in Kiew akkreditierten ausländischen Botschaftern, dass die Ukraine den Sicherheitsdialog mit der NATO fortsetzt.

Die Ukraine-Analysen werden mit Unterstützung durch die Otto-Wolff-Stiftung gemeinsam von der Forschungsstelle Osteuropa an der Universität Bremen und der Deutschen Gesellschaft für Osteuropakunde herausgegeben.

Die Meinungen, die in den Ukraine-Analysen geäußert werden, geben ausschließlich die Auffassung der Autoren wieder. Abdruck und sonstige publizistische Nutzung sind nach Rücksprache mit der Redaktion gestattet.

Redaktion und technische Gestaltung: Matthias Neumann, Heiko Pleines

Ukraine-Analysen-Layout: Cengiz Kibaroglu, Matthias Neumann

ISSN 1862-555X (c) 2007 by Forschungsstelle Osteuropa, Bremen

Forschungsstelle 0steuropa • Publikationsreferat • Klagenfurter Str. 3 • 28359 Bremen • Telefon: +49 421-218-7891 • Telefax: +49 421-218-3269

e-mail: publikationsreferat@osteuropa.uni-bremen.de • Internet-Adresse: www.ukraine-analysen.de 


\section{Lesetipp}

Arbeitspapiere und Materialien der Forschungsstelle Osteuropa ISSN 1616-7384

Nr. 81 - Januar 2007

Heiko Pleines $(\mathrm{Hg}$.$) :$

Das Comeback von Viktor Janukowitsch

Die innenpolitische Entwicklung in der Ukraine 2006

Inhaltsverzeichnis

Taras Kuzio

Die politische Landschaft vor den Parlamentswahlen .5

Kerstin Zimmer

Eine Region und ihre Partei. Die Partei der Regionen

als Donezker Elitenprojekt

Thomas Stiglbrunner

Die wichtigsten Parteien im Portrait.

Dokumentation

Das vorläufige amtliche Endergebnis der Parlamentswahl vom 26.3.2006

Chronik

Koalitionsverhandlungen und Regierungsbildung vom 26. März bis 6. September 2006.

Heiko Pleines

Demokratie lernen

Dokumentation

Die ursprüngliche Zusammensetzung der Regierung Janukowitsch .25

Kurzbiographien der Minister 27

Der Konflikt um die Entlassung des Außenministers

Ralf Wachsmuth

Ist Nascha Ukraina noch zu retten? Der schleichende Niedergang der Partei des Präsidenten....

Dokumentation

Präsident Viktor Juschtschenko zur politischen Lage ..... .35 


\section{Lesetipp}

\section{Abstracts mit Ukrainebezug aus dem gerade erschienenen Themenheft OSTEUROPA 2-3/2007 [Inklusion, Exklusion, Illusion. Konturen Europas: Die EU und ihre Nachbarn] \\ Das Heft kann bei der Redaktion OSTEUROPA erworben werden.}

osteuropa@dgo-online.org, Tel.: 030 / 30104582

\section{Arkadij Mošes}

Priorität gesucht

Die EU, Rußland und ihre Nachbarn

Die EU unterhält eine strategische Partnerschaft mit Rußland und will mit den Nachbarstaaten im Osten privilegierte Beziehungen unterhalten.

Zunehmend kollidieren die Interessen Rußlands und der EU im Nachbarschaftsraum. Moskau wertet die Nachbarschaftspolitik als Versuch, Rußland aus der Region zu drängen, und reagiert allergisch auf Demokratisierung. Doch wäre es falsch, wenn die EU ihr Engagement in diesem Raum verringert. Brüssel muß bereit sein, mehr Verantwortung zu übernehmen. Priorität sollten Demokratie und Marktwirtschaft, nicht aber eine geopolitische Umorientierung der Region haben. So könnten die Nachbarländer ihre Verbindungen mit Rußland aufrechterhalten. Und Rußland könnte der EU nicht länger unterstellen, eine Politik nach den Regeln des Nullsummenspiels zu betreiben.

\section{Egbert Jahn}

Ausdehnung und Überdehnung

Von der Integrationskonkurrenz zwischen Brüssel und Moskau zum Ende der europäischen Integrationsfähigkeit Rußland hat in den 1990er Jahren immer mehr sein Potential als alternatives Integrationszentrum zu EU und NATO verloren. Die Integrationskonkurrenz zwischen Brüssel und Moskau hat sich zu einer Konkurrenz um Mitgliedschaft in der EU und um privilegierte Beziehungen mit dem einzigen dynamischen, Prosperität, Sicherheit, politische Stabilität, Freiheiten und Demokratisierung versprechenden Integrationszentrum Brüssel reduziert. Gleichzeitig ist die Integrationskraft der EU begrenzt. Wirtschaftlich und institutionell wäre die EU zwar von einer Aufnahme der osteuropäischen Nachbarstaaten nicht überfordert. Politisch ist dies jedoch gegenwärtig nicht durchsetzbar. Die bilaterale Demokratisierungspolitik, die mit vagen Beitrittsversprechen operiert, sollte durch eine klare Regionalisierungspolitik ergänzt werden, die Demokratie, Rechtsstaatlichkeit und Marktwirtschaft in regionalen Zusammenschlüssen an der EU-Außengrenze fördert.

\section{Iris Kempe}

Zwischen Anspruch und Realität

Die Europäische Nachbarschaftspolitik

Mit ihrer Nachbarschaftspolitik verfolgt die EU ein hochgestecktes Ziel.

Sie will von der Barentssee bis zum Mittelmeer einen Ring befreundeter Staaten schaffen und so Sicherheit und Stabilität jenseits der EU-Außengrenzen garantieren. Die neuen Kooperationsangebote umfassen zahlreiche Politikfelder, schließen aber einen Beitritt zur EU aus. Die Nachbarschaftspolitik ist daher nur begrenzt dazu geeignet, Sicherheit und Stabilität in den Anrainerstaaten zu schaffen.

Clara M. O’Donnell, Richard G. Whitman

Das Phantom-Zuckerbrot

Die Konstruktionsfehler der ENP

Die EU will Stabilität, Demokratie, Rechtsstaatlichkeit und Marktwirtschaft in den Staaten an den EUAußengrenzen verbreiten. Um die Nachbarstaaten zu Reformen zu bewegen, bietet die EU ihnen in der Europäischen Nachbarschaftspolitik eine Reihe von Anreizen. Diese reichen jedoch bei weitem nicht aus. Der einzige Reformhebel, der wirken könnte, wäre eine Aussicht auf Mitgliedschaft in der EU. Zu diesem Zuckerbrot kann sich die EU jedoch bislang nicht entschließen. So rennt etwa die Ukraine einem Phantom hinterher. 


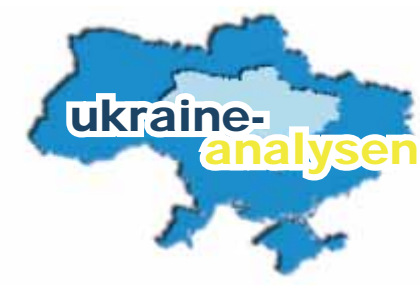

\section{Steffi Franke}

Die Grenze, die keine sein möchte

Exklusion und Inklusion an der EU-Ostgrenze Menschen aus dem Grenzgebiet warnen davor, daß an der Ostgrenze der EU neue Barrieren entstehen. Um dem entgegenzuwirken, ist grenzüberschreitende Zusammenarbeit wichtig. Doch Grenzen müssen nicht zwangsläufig Ausschluß bedeuten. Die Entwicklung am polnischen Abschnitt der neuen EU-Außengrenze zeigt dies. Im Nachbarschaftsprogramm spiegeln sich die Widersprüche des Grenzkonzepts. Kennzeichnend ist die Gleichzeitigkeit unvereinbarer Praktiken, wodurch neue Handlungsspielräume entstehen.

Ol'ga Šumylo

Draußen vor der Tür

Die ENP aus Sicht der Ukraine

Denjenigen Ukrainern, die ihr Land gerne in der EU sähen, gilt die Europäische Nachbarschaftspolitik als schlechter Ersatz. Auch Hoffnungen, die ENP werde Demokratisierung und Wirtschaftsreformen fördern, haben sich nicht erfüllt. Die Pläne der EU zur ENP-Reform geben aber diesen Hoffnungen neue Nahrung. Trotz mancher Defizite enthält das neue Konzept einige Punkte, die - sollten sie in die Tat umgesetzt werden - für die Ukraine eine stärkere Integration in die EU jenseits der Vollmitgliedschaft bedeuten könnten. Eine deutlichere Prioritätensetzung, klarere Anreize, mehr technische und finanzielle Unterstützung durch die EU und vor allem ein Ausbau der Wirtschafts- und Handelsbeziehungen durch einen „vertieften“ Freihandel wären die beste Gewähr für einen Wirtschaftsaufschwung und eine Verankerung der Ukraine in Europa.

\section{Lior Herman, Evgeny Finkel}

Zweierlei Maß

Die ENP-Aktionspläne: Israel und die Ukraine Die Ukraine und Israel spielen aus der Perspektive der EU in ihren Regionen eine Schlüsselrolle. Dieser Sonderstatus schlägt sich jedoch in den EU-Aktionsplänen für beide Länder unterschiedlich nieder. Während die EU Israel engere bilaterale Beziehungen anbietet und dessen Sicherheitsinteressen entgegenkommt, ist der EU-Ukraine-Aktionsplan weniger Zeugnis einer Wertegemeinschaft gleichberechtigter Partner als einseitiger Vorschriftenkatalog an die Adresse der Ukraine, ohne daß diese die Perspektive auf einen EU-Beitritt erhielte. Der Vergleich offenbart die teilweise Diskrepanz, die zwischen den postulierten Zielen der Europäischen Nachbarschaftspolitik und ihrer Realisierung in den Aktionsplänen besteht, sowie die Schwierigkeit, Mechanismen aus der EU-Erweiterung auf unterschiedliche Nichtkandidatenländer anzuwenden.

\section{Alexei Sekarev}

\section{Nachbarschaft in der Provinz}

\section{Erkundungen in der Westukraine}

Die Europäische Nachbarschaftspolitik hat besondere Implikationen für die grenznahen Gebiete der Nachbarstaaten. Am Beispiel der Ukraine zeigt sich, daß neben der Zentralregierung auch regionale und kommunale Verwaltungen, Industrie- und Handelskammern sowie NGOs Reformfähigkeit beweisen müssen, um die Wettbewerbsfähigkeit der Regionen zu erhöhen und um sich fit zu machen für die sektorale Einbindung in den Binnenmarkt und die Übernahme von Normen und Standards der EU. Diese Politik bietet eine Chance zur Modernisierung von Wirtschaft und Verwaltung in der westlichen Ukraine.

\section{Anita Szymborska}

\section{Freundliche EU-Grenze}

Anspruch und Realität der EU-Visapolitik Mit Sympathiebekundungen für Demokratiebewegungen in Osteuropa ist die EU stets schnell zur Stelle. Bei der Visapolitik hört die Freundschaft jedoch auf. So werden - dies zeigt ein Monitoring der Warschauer Stefan-Batory-Stiftung - Bürgern der Ukraine, aus Belarus, Moldova und Rußland trotz Erleichterungen auf dem Papier immer noch massive Hindernisse in den Weg gelegt, wenn sie sich um Visa für den Schengen-Raum bemühen. Die neuen EU-Mitgliedstaaten, allen voran Polen, sehen sich gezwungen, ihre liberale Visapolitik gegenüber ihren östlichen Nachbarn an die restriktivere Schengen-Gesetzgebung anzupassen. Auch ein neues Abkommen der EU mit der Ukraine über Visaerleichterungen bringt nur zweifelhafte Fortschritte.

\section{Natascha Wessel}

\section{Nonproliferationskooperation}

Europäisch-ukrainische Exportkontrolle wider die Verbreitung von Massenvernichtungswaffen Die Europäische Union sieht die Verbreitung von Massenvernichtungswaffen als große Sicherheitsbedrohung. Um die Proliferation einzudämmen, versucht sie, die Exportkontrollbehörden anderer Staaten zu stärken. In der Ukraine untersucht das von der EU beauftragte Bundesamt für Wirtschaft und Ausfuhrkontrolle das Außenwirtschaftsrecht, gibt Ratschläge zur Anpassung an europäische Standards und internationale Nonproliferationsregimes und schult Beamte und Unternehmer. 


\section{Lesehinweis}

\section{Kostenlose E-Mail-Dienste der Forschungsstelle Osteuropa}

\section{Russlandanalysen}

Die „Russlandanalysen“ bieten wöchentlich eine Kurzanalyse zu einem aktuellen Thema, ergänzt um Grafiken und Tabellen. Zusätzlich gibt es eine Wochenchronik aktueller politischer Ereignisse.

Abonnement unter: fsopr@uni-bremen.de

\section{Russian Analytical Digest}

Der Russian Analytical Digest bietet zweimal monatlich englischsprachige Kurzanalysen sowie illustrierende Daten zu einem aktuellen Thema.

Abonnement unter: http:// w w w.res.ethz.ch/ analysis/ rad/

\section{kultura. Russland-Kulturanalysen}

Die Russland-Kulturanalysen diskutieren in kurzen, wissenschaftlich fundierten, doch publizistisch-aufbereiteten Beiträgen signifikante Entwicklungen der Kultursphäre Russlands. Jede Ausgabe enthält zwei Analysen und einige Kurztexte bzw. Illustrationen. Erscheinungsweise: monatlich, in je einer deutschen und englischen Ausgabe.

Abonnement unter: fsopr@uni-bremen.de

\section{Ukraine-Analysen}

Die Ukraine-Analysen bieten zweimal monatliche eine Kurzanalyse zu einem aktuellen Thema aus Politik, Wirtschaft oder Kultur, ergänzt um Grafiken und Tabellen. Zusätzlich gibt es eine Chronik aktueller Ereignisse.

Abonnement unter: fsopr@uni-bremen.de

\section{Polen-Analysen}

Die Polen-Analysen bieten zweimal monatliche eine Kurzanalyse zu einem aktuellen Thema aus Politik, Wirtschaft oder Kultur, ergänzt um Grafiken und Tabellen. Zusätzlich gibt es eine Chronik aktueller Ereignisse.

Abonnement unter: http:// w w w.polen-a nalysen.de

\section{Bibliographische Dienste}

Die vierteljährlich erscheinenden Bibliographien informieren über englisch- und deutschsprachige Neuerscheinungen zu Polen, Russland, Tschechischer und Slowakischer Republik sowie zur Ukraine. Erfasst werden jeweils die Themenbereiche Politik, Außenpolitik, Wirtschaft und Soziales.

Abonnement unter: fsopr@uni-bremen.de

\section{FSO-Fernsehtipps}

Die „FSO-Fernsehtipps“ bieten zweiwöchentlich einen Überblick über Sendungen mit Bezug auf Ost- bzw. Ostmitteleuropa im deutschsprachigen Kabelfernsehen. Vorrangig erfaßt werden Spiel- und Dokumentarfilme aus und über osteuropäische Länder. Der Schwerpunkt liegt auf der Sowjetunion und ihren Nachfolgestaaten (vor allem Russland), Polen, Tschechien, Slowakei und DDR.

Abonnement unter: fsopr@uni-bremen.de 